\title{
Effects of Variety, Spacing and Nitrogen Application on Chickpea (Cicer arietinum) Growth and Yield in Embu County, Kenya
}

\author{
Judith N. K. Wafula ${ }^{1, *}\left(\mathbb{D}\right.$, Fredrick N. Mugendi ${ }^{1}$, Paul N. Nthakanio ${ }^{1}$, \\ Jeremiah O. Mosioma ${ }^{2}$, Charles A.N. Onyari ${ }^{2}$
}

${ }^{1}$ Department of Agricultural Resources Management, University of Embu, P.O. Box 6-60100, Embu, Kenya

${ }^{2}$ Department of Land and Water Management, University of Embu, P.O. Box 6-60100, Embu, Kenya

Received - November 18, 2021; Revision - February 02, 2022; Accepted - February 20, 2022

Available Online - February 28, 2022

DOI: http://dx.doi.org/10.18006/2022.10(1).32.47

\begin{tabular}{l} 
KEYWORDS \\
Chickpea \\
Growth \\
Spacing \\
Starter nitrogen fertilizer \\
Interactions \\
Yield \\
\hline
\end{tabular}

\begin{abstract}
Chickpea is a pulse crop that is rich in proteins and helps in fulfilling the requirement of protein for vegetarian people thus yield maximization needed. This study aimed to determine the effects of spacing, nitrogen rates, and their interactive effects on the growth and productivity of the selected chickpea varieties. Field experiments were conducted at Mwea, Kenya between 2017 and 2018 involving four varieties (Saina K, Mwanza 2, Chaina I and Chaina III) at a spacing of $50 \times 10 \mathrm{~cm}, 50 \times 20 \mathrm{~cm}$, and $50 \times 30 \mathrm{~cm}$, and starter nitrogen-fertilizer application rates $0 \mathrm{~kg}, 30 \mathrm{~kg}, 60 \mathrm{~kg}$, and $90 \mathrm{~kg} \mathrm{ha}^{-1}$. A split-split plot design arranged in a $4 \times 3 \times 4$ layout was used in the current study. Data related to plant height, biomass, grain yield, and harvest index were collected and subjected to statistical analysis by GLM in SAS 9.4 computer software. Variations occurred in measured traits like the height of crops (34.81$38.00 \mathrm{~cm})$, biomass yield $\left(3.31-8.08 \mathrm{tha}^{-1}\right)$, seed yield $\left(0.14\right.$ to $\left.1.9 \mathrm{tha}^{-1}\right)$, and percent harvest index $(5-$ $45 \%$ ) was reported. Mwanza 2 expressed the highest plant height, biomass, and grain yield. The highest plant biomass was obtained under $50 \times 10 \mathrm{~cm}$ spacing, while the highest grain yield weight was reported under $50 \times 30 \mathrm{~cm}$ spacing $\times 60 \mathrm{~kg} \mathrm{~N} \mathrm{ha}^{-1}$. From the results of the study, it can be concluded that the highest enhanced growth and productivity of chickpea were realized at interactions of Mwanza $2 \times 50 \times 10 \mathrm{~cm} \times 90 \mathrm{~kg} \mathrm{ha}^{-1}$ nitrogen rate in the study area.
\end{abstract}

* Corresponding author

E-mail: wafula.judith@embuni.ac.ke (Judith N.K. Wafula)

Peer review under responsibility of Journal of Experimental Biology and Agricultural Sciences.

Production and Hosting by Horizon Publisher India [HPI] (http://www.horizonpublisherindia.in/).

All rights reserved.
All the articles published by Journal of Experimental Biology and Agricultural Sciences are licensed under a Creative Commons Attribution-NonCommercial 4.0 International License Based on a work at www.jebas.org.

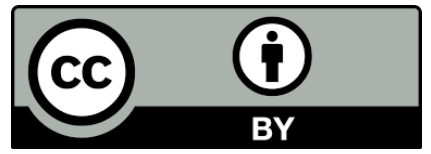




\section{Introduction}

Chickpea (Cicer arietinum) belonging to the Fabaceae family, is an important legume crop that is rich in protein. It has numerous agricultural benefits that made this crop an excellent crop rotation and intercrop mainly with cereal crops. It plays an important role in improving soil health and breaking some diseases and pest cycles (Raimi et al. 2017; Li et al. 2019; Fikre et al. 2020). The inclusion of chickpea in rotation to non-host plants provides sufficient time for decomposition of infested crop residues and reduces the viability of the pathogens (Berrada et al. 2007). Chickpea enhances soil fertility by its biological nitrogen fixation ability. It is estimated to fix up to $140 \mathrm{~kg} \mathrm{~N} \mathrm{ha}^{-1}$ from the air through symbiotic nitrogen fixation and meets approximately $80 \%$ of soil N requirement (Hossain et al. 2016; Schilt-van et al. 2020) In Kenya chickpea crop grows as an intercrop to improve soil fertility and maximize yield (Ogola et al. 2013; Ndukhu et al. 2017). Additionally, the crop act as biocontrol of grassy weeds when used as an intercrop with cereals by covering the ground and smothering the weeds (Banik et al. 2006; Mousavi 2019,). This crop also has the ability of drought tolerance and can survive under scanty rainfall conditions with optimum precipitation of 152 $254 \mathrm{~mm}$ and temperatures range of $18-29^{\circ} \mathrm{C}$ (McVay and Crutcher 2011; Devasirvatham and Tan 2018; Kaloki et al. 2019a). Its deep root system penetrates deeper soil horizons for moisture (Onyari et al. 2010; Arif et al. 2021). The legumes are a good source of proteins, dietary fiber, vitamins A, C, E, folate, and minerals (Wallace and Zelman 2016; Muehlbauer and Sarker 2017). This crop is used as human food and surplus sold to earn income, while its remnants are used as fodder for animals.

Worldwide, the chickpea crop has the third position after common bean (Phaseolus vulgaris) and field pea (Pisum sativum) Internationally, Asia, Europe, America, and Australia are key chickpea producers (Wafula et al. 2021) and accounting 95\% of the world's chickpea production (Merga and Haji 2019). The global chickpea production steadily increased from 7.7million tonnes to over 14.2 million tonnes from 2006 to 2019 (FAOSTAT, 2019). According to Merga and Haji (2019), over 1.3 million tons of chickpea from major producers enter the market to supplement the needs of countries unable to meet their production demands. A number of these deficient countries are in Africa where the demand is higher than the supply (Maya and Maphosa 2020). Major chickpea growing areas of Africa are located in the North, West, and Eastern Africa (Maya and Maphosa 2020). These countries contribute about $63 \%$ of total African chickpea production (Abebe and Debebe 2020).

In Kenya, chickpea is ranked fourth after common bean, field pea, and cowpeas (Mallu 2015). Its production is dominant in the dryland regions that include parts of the Rift Valley and Eastern regions of Kenya including Mwea, Embu County. Farming systems within Mwea, Karaba location, are rain-fed with limited use of inorganic fertilizers. Pellic vertisol is the dominant soil in the area. Crops grown in the region are drought-tolerant, such as field pea, cowpea, chickpea, and sorghum (Wafula et al. 2021). The annual chickpea productivity in Kenya is low with an average yield of $357 \mathrm{~kg} \mathrm{ha}^{-1}$ (FAOSTAT, 2019). The limited performance of crops might be due to selection from a narrow range of landraces (Rao et al. 2012). The main varieties used in Kenya are (Desi 1), ICCV 00108, ICCV 92944 (Desi II), ICCV 97126 (Desi III), and Saina K, ICCV 00305 (Kabuli) (Gaur et al. 2010). Drastic variation in soils and differences in agro-ecological zones contribute to poor crop performance (Jaetzold and Schmidt 1982). In addition, the landrace varieties may not be adaptable to all agroecological zones thus, necessitating test performance to determine suitability in growth areas. It, therefore, becomes needful to test the performance of these varieties in specific areas.

Another factor that affects the chickpea performance is sowing spacing due to influence in light interception hence photosynthesis. Spacing affects the number of soil nutrients and water that an individual plant can access (Khan et al. 2010). Initial spacing of $30 \times 10 \mathrm{~cm}$ tested in Kenya gave yields between 1200 to $1500 \mathrm{~kg} \mathrm{ha}^{-1}$ (Gaur et al. 2010). Due to changing climate and soil fertility, revision of this spacing is necessary to enhance the productivity of the crops (Kamithi et al. 2009; Ngetich et al. 2014). Agajie (2014) reported variation in chickpea yields from $1219 \mathrm{~kg} \mathrm{ha}^{-1}(30 \mathrm{x} 10 \mathrm{~cm})$, $1134 \mathrm{~kg} \mathrm{ha}^{-1}(30 \mathrm{x} 15 \mathrm{~cm}), 1049 \mathrm{~kg} \mathrm{ha}^{-1}(40 \times 10 \mathrm{~cm}), 1019 \mathrm{~kg} \mathrm{ha}^{-1}$ $(50 \times 5 \mathrm{~cm}), 733 \mathrm{~kg} \mathrm{ha}^{-1}(50 \times 15 \mathrm{~cm})$ and $1088 \mathrm{~kg} \mathrm{ha}^{-1}(20 \times 10 \mathrm{~cm})$ under various spacing. Further, Mallu (2015) also reported that in Kenya biomass of chickpea varieties ranges from 2.9 to 7.4 tonnes per hectare under different spacing growth conditions. Similarly, Kerina et al. (2017) also obtained higher biomass and grain yield for various crops under $45 \times 20 \mathrm{~cm}$ spacing across various regions.

Over the years, soil conditions have continuously deteriorated due to nutrient mining by continuous cropping without replenishing the nutrients, nutrient export (crop residues transferred to other areas), overcropping, and mono-cropping (Kiboi et al. 2019). In addition, erosion due to erratic and unreliable rainfall and prolonged droughts in arid areas also contributes to nitrogen losses (Kimiti et al. 2009). Cumulatively this contributes to reduced chickpea productivity. Other factors that have contributed to low $\mathrm{N}$ in soil include leaching due to floods, increased human activity such as rampant bush clearing and burning (Kerina et al. 2017). Accumulations of these activities have led to the reduction of soil $\mathrm{N}$ to about $0.12 \%$ of the average plant requirement in semi-arid regions of Kenya (Wafula et al. 2021). Although chickpea can fix nitrogen $(\mathrm{N})$, soils with $\mathrm{N}$ deficiency require small doses of starter $\mathrm{N}$-fertilizer at planting time to promote early growth (Lemma et al. 2013; Dar et al. 2021). Thus, legumes also need $\mathrm{N}$-fertilizer 
application to boost their productivity (Li et al. 2016). Variability in N-levels, spacing, and narrow chickpea germplasm in growing areas of Kenya, may be responsible for depressed yields of chickpea in Embu county. This study, therefore, investigates the effect of variety, plant spacing, level of nitrogenous fertilizer at sowing time, and their interactions on plant growth and grain yield of chickpea in Embu County.

\section{Materials and methods}

\subsection{Site description and environment}

Field experiments were conducted in the Karaba area of Mwea Ward, Mbeere South Sub-County, Embu County, Kenya (Figure 1). The study site lies at coordinates $0^{\circ} 46^{\prime} 14.4822^{\prime} \mathrm{S}$ and $37^{\circ}$
22'23.79324'E and an altitude of 980 meters above sea level. Karaba receives bimodal rainfall patterns with annual rainfall amounts ranging from 600 to700 $\mathrm{mm}$ according to MIAD weather data, resulting in growing seasons between March to May and October to December (Wafula et al. 2021). In addition, the average minimum and maximum temperatures fluctuate between $18-20^{\circ} \mathrm{C}$ and $19-24^{\circ} \mathrm{C}$ (KMD 2017) respectively. However, over the cropping period, precipitation recorded was 264.3mm (LR2017), 408mm (SR2017), and 787.1mm (LR2018), while minimum and maximum temperatures recorded ranged from $13-16^{\circ} \mathrm{C}$ and $22-29^{\circ} \mathrm{C}$ respectively (Figure 2). Site selection for field trials was due to the uniformity of the soils and environmental conditions among many farmers in the local area (Figure 1) (Wafula et al. 2021).

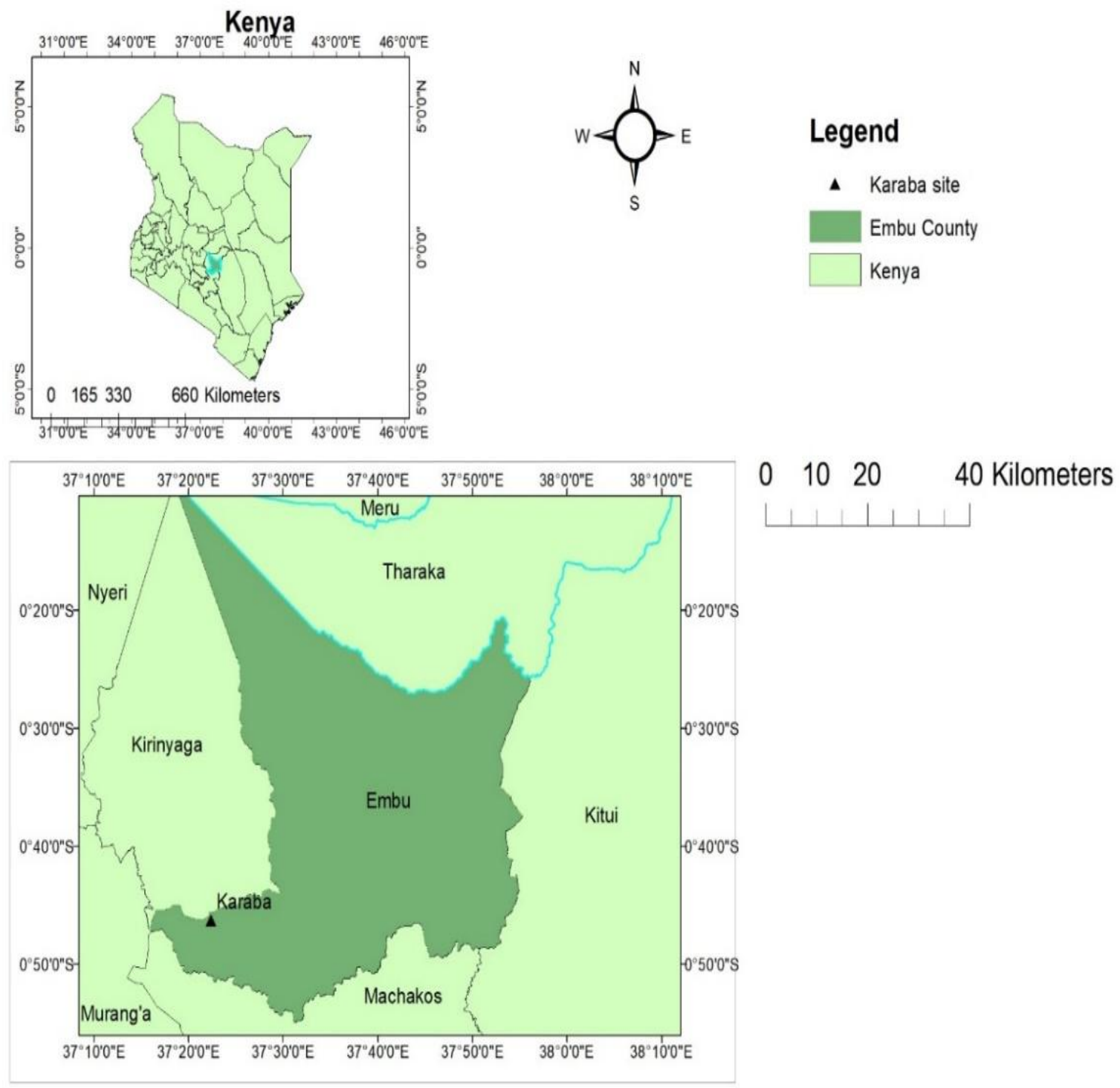

Figure 1 Map of the study area. Karaba. Explain the scale

Journal of Experimental Biology and Agricultural Sciences http://www.jebas.org 

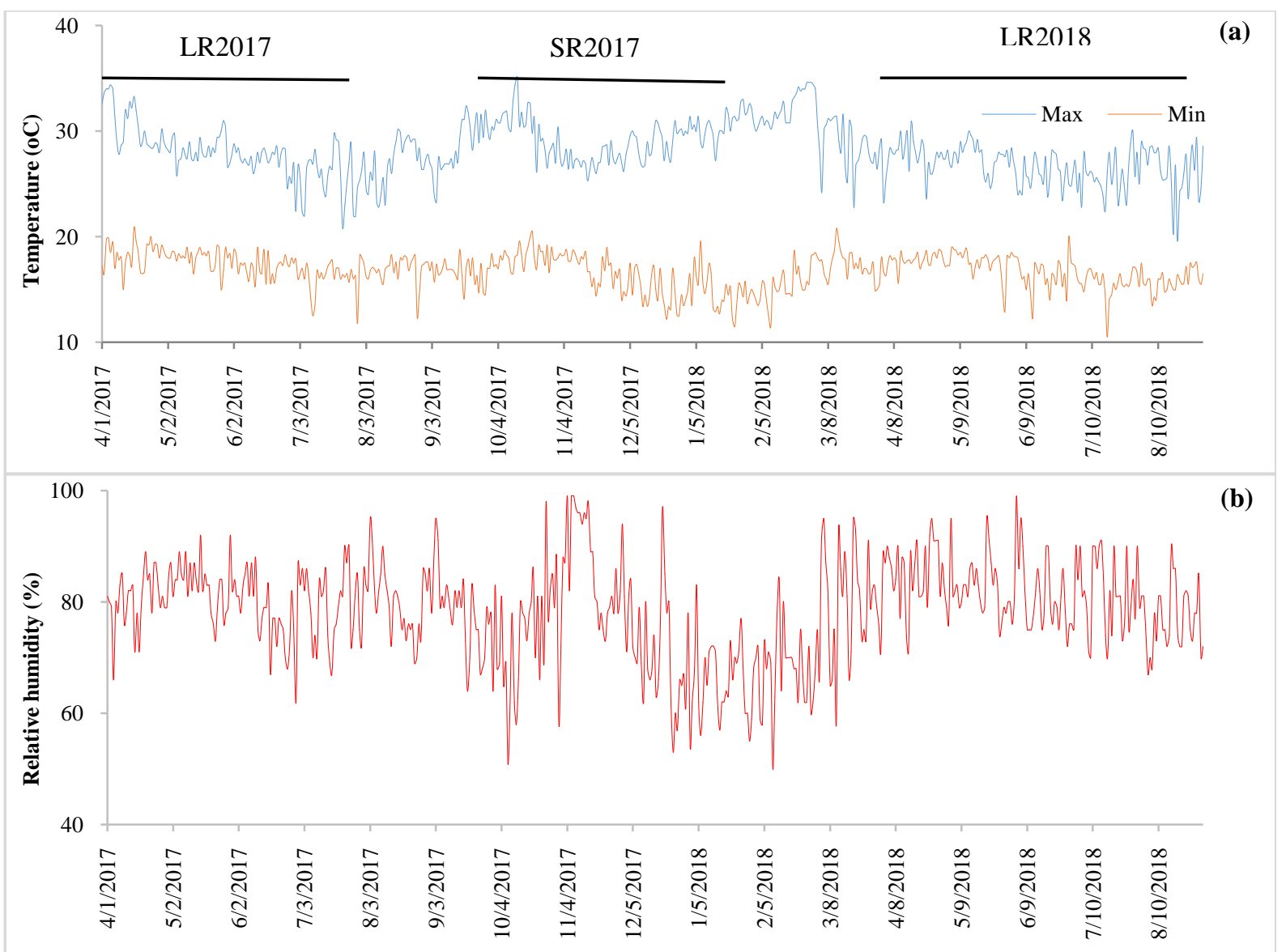

(b)

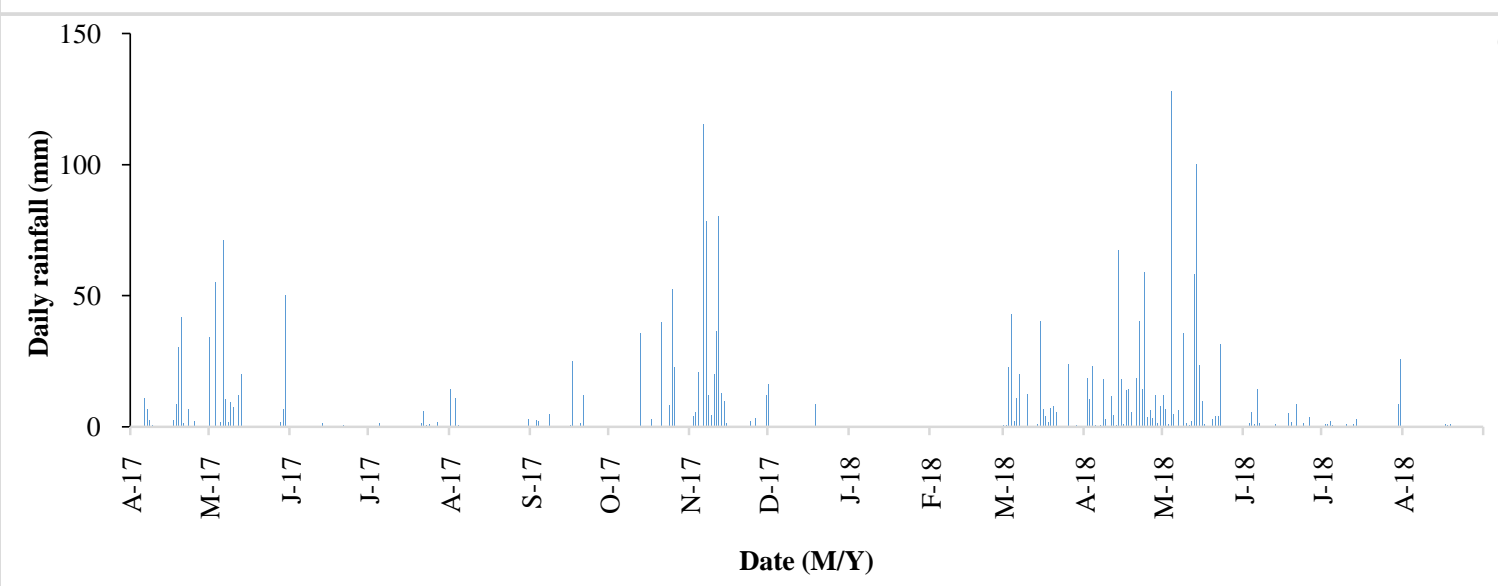

Figure 2 Daily weather data a) maximum and minimum temperature $\left({ }^{\circ} \mathrm{C}\right)$, b) relative humidity $(\%)$ and c) rainfall $(\mathrm{mm})$. Max indicates maximum and min indicates minimum. LR is the long rains and SR is the short rains. Source: (KMD 2017; KMD 2018). In X-axis first leter stand for month and $17=2017$ while $18=2018$.

\subsection{Setting the field trials}

The experiment was a split-split plot design (SSPD) of a $4 \times 3 \times 4$ factorial arrangement. Each plot have a $3 \times 3 \mathrm{~m}$ size and had six rows of the variety having 30,15 , and 10 planting holes per row concerning spacing used. Accordingly, the arrangement of factors within the plot was chickpea variety (main plot), spacing (subplot), and inorganic fertilizer (sub-sub plot). Land preparations were done in February 2017, before the onset of long rain using a tractor-mounted with a disc plow to remove hardpans, followed by 
harrowing using a disc harrow for the first season (LR2017), then leveling using a hoe (jembe). A hoe was used in subsequent seasons in September for season 2 (SR2017) and in February for season 3 (LR2018) for all field operations.

Sourcing of planting materials was from Kenya Seed Company, Nakuru, while di-ammonium phosphate (DAP) was collected locally from Mwea. DAP fertilizer is recommended for the area by the Ministry of Agriculture, Kenya (MoA, 2013). Two seeds were sown per hole at a spacing of $50 \times 10 \mathrm{~cm}, 50 \times 20 \mathrm{~cm}$, and $50 \times 30 \mathrm{~cm}$ that were thinned two weeks after germination to leave one seedling per hole and enable obtaining the required plant population of 200000, 100000, and 70000 per hectare Application of fertilizer was carried out at planting time, using four rates of $\mathrm{N}_{0}\left(0 \mathrm{~kg} \mathrm{~N} \mathrm{ha}^{-1}\right), \mathrm{N}_{30}\left(30 \mathrm{~kg} \mathrm{~N} \mathrm{ha}^{-1}\right), \mathrm{N}_{60}(60 \mathrm{~kg} \mathrm{~N}$ ha $\left.{ }^{1}\right)$, and $\mathrm{N}_{90}\left(90 \mathrm{~kg} \mathrm{~N} \mathrm{ha}{ }^{-1}\right)$. Application of pre-emergence herbicide glyphosate @ 480g/L (Weedall) and organo-phosphate @ 26.25EC (Sumithion super) pesticide was carried out after planting to control weeds and crickets correspondingly. The crop was harvested when $90 \%$ of leaves had dried and dropped on the ground.

\subsection{Data collection}

Within each plot, a $1.5 \times 2 \mathrm{~m}$ section was purposively marked, and from this, data were collected on plant height and total biomass every after two weeks at the stages of 56,70, and 84 DAS, while grain yield data were only recorded at 84DAS. For the estimation of biomass, three plants were uprooted, bagged, and transported to the University of Embu and oven-dried at $68^{\circ} \mathrm{C}$ for 72 hours The weights were taken in grams using an electronic balance (Model BA2204B, Machine number 040683), and an average per plant was determined. For grain yield, three plants were manually harvested and sun-dried to a constant weight, and the weight was recorded at $13 \%$ moisture content using equation 1 given by Ngetich et al. (2014). Harvest index was determined by calculating the ratio of average grain yield to biomass per plant, and then multiplied by 100 (Patil et al. 2021). Standard management practices were carried out when necessary (Kalungu and Harris 2013).

Grain yield $(t h a-1)=$ Grain weight $* \frac{S M C \%}{A M C \%}$

Equation 1

Where AMC means actual moisture content of sample obtained using a moisture meter, SMC means Standard moisture content $(13 \%)$.

Data were analyzed using the Generalized Linear Model (GLM), (Rutherford 2011) in SAS 9.4 software (SAS 2015). Mean separation was done using Turkey's honestly significant difference
(HSD) test and Duncan's multiple range test (DMRT).for interactions means at a $95 \%$ confidence level.

\section{Results}

\subsection{Chickpea plant height}

Plant height significantly varied $(\mathrm{P}<0.05)$ across the seasons (Table 1). At 56 days after sowing (DAS) Chaina I and Chaina III had the highest plant height, while between 70 and 84 DAS Mwanza 2 had the highest plant height in both the long and the short rainfall (Table 1). In addition, the effect of spacing was significantly high at 56DAS $(50 \times 10 \mathrm{~cm})$ in LR2017 compared to other spacing. During SR2017, both 50x10cm and 50x30 $\mathrm{cm}$ had a similar height of $34.9 \mathrm{~cm}$ and $34.8 \mathrm{~cm}$ respectively, while $50 \times 20 \mathrm{~cm}$ scored the least $(33.8 \mathrm{~cm})$. Nitrogen fertilizer rates $\mathrm{N}_{0}$ and $\mathrm{N}_{30}$ had significantly high differences compared to $\mathrm{N}_{60}$ and $\mathrm{N}_{90}$ in plant height at 56 and 70DAS apart from the LR2017 growth condition (Table 1). At 84DAS, $\mathrm{N}_{90}$ was significantly superior in height than other N-rates over the LR2017 growth conditions. However, in SR2017 and LR2018 plant growth was significantly reduced (Table 1).

3.2 Effects of imposed treatment on dry biomass, grain yield, and harvest index

Results of the study suggested that Saina K and Mwanza 2 have the highest biomass under LR2017 and SR2017 growth conditions compared to Chaina I and Chaina III (Table 2). The biomass decreased as spacing increased from $50 \times 10 \mathrm{~cm}\left(8.16 \mathrm{t} \mathrm{ha}^{-1}\right)$ in LR2017 to $3.07 \mathrm{t} \mathrm{ha}^{-1}$ in $50 \times 30 \mathrm{~cm}$. Additionally, an application rate of $\mathrm{N}_{60}$ and $\mathrm{N}_{90}$ had a significant effect $(\mathrm{P}<0.05)$ on biomass during LR2017 and SR2017 compared to the other N-rates (Table 2).

Genotype Mwanza 2 had a significantly higher grain yield weight than other varities in LR2017, but Saina K registered significantly higher grains yield than the others in the cropping season of SR2017. However, under LR2018, Saina K and Mwanza 2 had significantly higher grain yield than the other two varieties. Spacing of 50x30cm gave the lowest grain yields in SR2017 but yielded better than other spacing in LR2017 although, no significant difference was reported in the yield in LR2018. Under the LR2017 and LR2018 growth season, $\mathrm{N}_{60}$ had the highest grain yield of $1.59 \mathrm{t} \mathrm{ha}^{-1}$ and $2.00 \mathrm{t} \mathrm{ha}^{-1}$ respectively (Table 2). In LR2017, Mwanza 2 (45\%) gave the highest harvest index but it was not significantly different from Chaina I (43\%) variety, whereas, in SR2017, Saina K performed better than all the other varieties. Spacing of $50 \times 30 \mathrm{~cm}$ posted a better harvest index in SR2017 and LR2018. However, in LR2017, spacing of 50x20cm posted the highest harvest index but it was not significantly different from $50 \times 30 \mathrm{~cm}$. Harvest index was also significantly 
Table 1 Plant height as influenced by variety, spacing and nitrogen application rate during the three cropping seasons in Mwea

\begin{tabular}{|c|c|c|c|c|c|c|c|c|c|}
\hline${ }^{1}$ Treatment & \multicolumn{3}{|c|}{ Height 56 DAS $(\mathrm{cm})$} & \multicolumn{3}{|c|}{ Height 70 DAS (50\% maturity for Chaina varieties) } & \multicolumn{3}{|c|}{ Height 84 DAS (50\% maturity for Mwanza 2 \& Saina K)) } \\
\hline Variety & LR $17^{2}$ & SR 17 & LR 18 & LR 17 & SR 17 & LR 18 & LR 17 & SR 17 & LR 18 \\
\hline Saina K & $33.8^{\mathrm{b}} \pm 0.30$ & $32.4^{\mathrm{b}} \pm 0.48$ & $32.4^{\mathrm{c}} \pm 0.45$ & $35.1^{\mathrm{a}} \pm 0.30$ & $34.6^{\mathrm{C}} \pm 0.48$ & $34.1^{\mathrm{c}} \pm 0.46$ & $35.8^{\mathrm{b}} \pm 0.24$ & $36.7^{\mathrm{b}} \pm 0.35$ & $36.39^{\mathrm{b}} \pm 0.35$ \\
\hline Mwanza 2 & $34.6^{\mathrm{a}} \pm 0.36$ & $34.0^{\mathrm{b}} \pm 0.68$ & $34.0^{\mathrm{b}} \pm 0.68$ & $35.7^{\mathrm{a}} \pm 0.38$ & $37.5^{\mathrm{a}} \pm 0.49$ & $36.3^{\mathrm{a}} \pm 0.27$ & $37.7^{\mathrm{a}} \pm 0.47$ & $38.0^{\mathrm{a}} \pm 0.47$ & $37.80^{\mathrm{a}} \pm 0.41$ \\
\hline Chaina I & $33.8^{\mathrm{b}} \pm 0.22$ & $36.8^{\mathrm{a}} \pm 0.40$ & $35.6^{\mathrm{a}} \pm 0.29$ & $35.4^{\mathrm{a}} \pm 0.31$ & $36.1^{\mathrm{c}} \pm 0.44$ & $35.5^{\mathrm{a}} \pm 0.32$ & $36.4^{\mathrm{b}} \pm 0.28$ & $35.1^{\mathrm{c}} \pm 0.38$ & $36.04^{\mathrm{b}} \pm 0.27$ \\
\hline Chaina III & $34.6^{\mathrm{a}} \pm 0.29$ & $34.8^{\mathrm{b}} \pm 0.35$ & $34.7^{\mathrm{ab}} \pm 0.39$ & $34.8^{\mathrm{a}} \pm 0.45$ & $35.6 b^{c} \pm 0.45$ & $35.1^{\mathrm{c}} \pm 0.31$ & $35.8^{\mathrm{b}} \pm 0.33$ & $34.8^{\mathrm{c}} \pm 0.55$ & $35.50^{\mathrm{b}} \pm 0.31$ \\
\hline \multicolumn{10}{|c|}{ Spacing } \\
\hline $50 \times 10 \mathrm{~cm}$ & $34.8^{\mathrm{a}} \pm 0.20$ & $34.9^{\mathrm{a}} \pm 0.20$ & $34.5^{\mathrm{a}} \pm 0.20$ & $35.87^{\mathrm{a}} \pm 0.21$ & $35.7^{\mathrm{a}} \pm 0.22$ & $35.2^{\mathrm{a}} \pm 0.21$ & $36.4^{\mathrm{b}} \pm 0.19$ & $35.0^{\mathrm{b}} \pm 0.19$ & $35.92^{\mathrm{b}} \pm 0.20$ \\
\hline $50 \times 20 \mathrm{~cm}$ & $33.4^{\mathrm{b}} \pm 0.18$ & $33.8^{\mathrm{b}} \pm 0.19$ & $33.8^{\mathrm{a}} \pm 0.24$ & $34.9^{b} \pm 0.19$ & $35.7^{\mathrm{a}} \pm 0.20$ & $35.2^{\mathrm{a}} \pm 0.21$ & $35.7^{\mathrm{C}} \pm 0.19$ & $36.4^{\mathrm{a}} \pm 0.20$ & $36.24^{\mathrm{b}} \pm 0.20$ \\
\hline $50 \times 30 \mathrm{~cm}$ & $34.2^{\mathrm{b}} \pm 0.25$ & $34.8^{\mathrm{a}} \pm 0.49$ & $34.2^{\mathrm{a}} \pm 1.01$ & $35.0^{\mathrm{b}} \pm 0.21$ & $36.4^{\mathrm{a}} \pm 0.45$ & $35.3^{\mathrm{a}} \pm 1.01$ & $37.2^{\mathrm{a}} \pm 0.21$ & $37.1^{\mathrm{a}} \pm 0.46$ & $37.14^{\mathrm{a}} \pm 1.08$ \\
\hline \multicolumn{10}{|c|}{ Nitrogen } \\
\hline $\mathrm{N}_{0}$ & $34.3^{\mathrm{a}} \pm 0.40$ & $33.5^{\mathrm{b}} \pm 0.62$ & $33.8^{\mathrm{b}} \pm 0.43$ & $35.2^{\mathrm{a}} \pm 0.35$ & $35.3^{\mathrm{bc}} \pm 0.42$ & $34.6^{\mathrm{b}} \pm 0.42$ & $35.6^{\mathrm{c}} \pm 0.20$ & $36.2^{\mathrm{ab}} \pm 0.43$ & $35.48^{\mathrm{b}} \pm 0.30$ \\
\hline $\mathrm{N}_{30}$ & $34.0^{\mathrm{a}} \pm 0.42$ & $33.5^{\mathrm{b}} \pm 0.37$ & $33.7^{\mathrm{b}} \pm 0.25$ & $34.9^{\mathrm{a}} \pm 0.52$ & $35.1^{\mathrm{c}} \pm 0.26$ & $34.9^{\mathrm{b}} \pm 0.41$ & $36.3^{\mathrm{cb}} \pm 0.54$ & $35.5^{\mathrm{b}} \pm 0.25$ & $36.29^{\mathrm{ab}} \pm 0.50$ \\
\hline $\mathrm{N}_{60}$ & $34.0^{\mathrm{a}} \pm 0.29$ & $35.9^{\mathrm{a}} \pm 0.27$ & $34.4^{\mathrm{a}} \pm 0.65$ & $35.0^{\mathrm{a}} \pm 0.32$ & $36.4^{\mathrm{ab}} \pm 0.24$ & $35.3^{\mathrm{ab}} \pm 0.45$ & $36.4^{\mathrm{b}} \pm 0.35$ & $36.2^{\mathrm{ab}} \pm 0.31$ & $36.70^{\mathrm{a}} \pm 0.54$ \\
\hline $\mathrm{N}_{90}$ & $34.4^{\mathrm{a}} \pm 0.27$ & $35.1^{\mathrm{a}} \pm 0.41$ & $34.9^{\mathrm{a}} \pm 0.36$ & $35.8^{\mathrm{a}} \pm 0.42$ & $36.9^{\mathrm{a}} \pm 0.53$ & $36.2^{\mathrm{a}} \pm 0.35$ & $37.4^{\mathrm{a}} \pm 0.31$ & $36.6^{\mathrm{a}} \pm 0.44$ & $37.26^{\mathrm{a}} \pm 0.42$ \\
\hline
\end{tabular}

Treatments (Variety, Spacing and Nitrogen). Cropping seasons: [LR17 (long rains 2017), SR (short rains 2017) and LR18 (long rains 2018)]. Nitrogen rates: [N $\mathrm{N}_{0}\left(0 \mathrm{~kg} \mathrm{~N}^{-1}{ }^{-1}\right)$; $\mathrm{N}_{30}\left(30 \mathrm{~kg} \mathrm{~N}\right.$ ha $\left.{ }^{-1}\right) ; \mathrm{N}_{60}$ $\left(60 \mathrm{~kg} \mathrm{~N} \mathrm{ha}^{-1}\right)$ and $\left.\mathrm{N}_{90}\left(90 \mathrm{~kg} \mathrm{~N} \mathrm{ha}^{-1}\right)\right]$. Sources of interactions: Variety (V), Spacing (S), N (Nitrogen), VS (Variety x Spacing), VN (Variety x Nitrogen), SN (Spacing x Nitrogen) and VSN (Variety x Spacing $x$ Nitrogen). Means with same letter in a column are not significantly different at $\mathrm{P} \leq 0.05$; Ns means not significant at $\mathrm{P} \leq 0.05$.

Table 2 Total biomass, grain yield and harvest Index as influenced by variety, spacing and nitrogen application rate

\begin{tabular}{|c|c|c|c|c|c|c|c|c|c|}
\hline Treatment $^{1}$ & \multicolumn{3}{|c|}{ Total biomass $\left(\mathrm{t} \mathrm{ha}^{-1}\right)$} & \multicolumn{3}{|c|}{ Grain yield $\left(\mathrm{t} \mathrm{ha}^{-1}\right)$} & \multicolumn{3}{|c|}{ Harvest index (HI\%) } \\
\hline Variety & $\mathrm{LR}_{2017^{2}}$ & SR2017 & LR2018 & LR2017 & SR2017 & LR2018 & LR2017 & SR2017 & LR2018 \\
\hline Saina K & $6.58^{\mathrm{a}} \pm 0.64$ & $8.08^{\mathrm{a}} \pm 0.54$ & $5.12^{\mathrm{b}} \pm 0.56$ & $1.49^{\mathrm{b}} \pm 0.09$ & $0.87^{\mathrm{a}} \pm 0.06$ & $1.91^{\mathrm{a}} \pm 0.11$ & $33^{\mathrm{c}} \pm 3.50$ & $12^{\mathrm{a}} \pm 0.65$ & $45 \mathrm{a} \pm 1.56$ \\
\hline Mwanza 2 & $6.29^{\mathrm{a}} \pm 0.56$ & $7.74^{\mathrm{a}} \pm 0.48$ & $7.13^{\mathrm{a}} \pm 0.30$ & $1.92^{\mathrm{a}} \pm 0.15$ & $0.47^{\mathrm{b}} \pm 0.06$ & $1.95^{\mathrm{a}} \pm 0.16$ & $45^{\mathrm{a}} \pm 5.34$ & $6^{\mathrm{b}} \pm 0.55$ & $40 \mathrm{a} \pm 3.06$ \\
\hline Chaina I & $4.24^{\mathrm{b}} \pm 0.25$ & $3.31^{\mathrm{c}} \pm 0.25$ & $4.29^{\mathrm{bc}} \pm 0.19$ & $1.55^{\mathrm{b}} \pm 0.12$ & $0.14^{\mathrm{c}} \pm 0.01$ & $1.23^{\mathrm{b}} \pm 0.13$ & $43^{\mathrm{a}} \pm 4.72$ & $5^{\mathrm{b}} \pm 0.70$ & $40 \mathrm{a} \pm 3.08$ \\
\hline Chaina III & $3.68^{\mathrm{c}} \pm 0.31$ & $4.38^{\mathrm{b}} \pm 0.25$ & $4.24^{\mathrm{c}} \pm 0.28$ & $1.21^{\mathrm{c}} \pm 0.08$ & $0.19^{\mathrm{c}} \pm 0.01$ & $1.07^{\mathrm{b}} \pm 0.25$ & $38^{\mathrm{b}} \pm 3.04$ & $5^{\mathrm{b}} \pm 0.40$ & $35 \mathrm{a} \pm 3.46$ \\
\hline \multicolumn{10}{|c|}{ Spacing } \\
\hline $50 \times 10 \mathrm{~cm}$ & $8.19^{\mathrm{a}} \pm 0.26$ & $8.00^{\mathrm{a}} \pm 0.26$ & $7.51^{\mathrm{a}} \pm 0.24$ & $1.15^{\mathrm{c}} \pm 0.06$ & $0.50^{\mathrm{a}} \pm 0.07$ & $1.49^{\mathrm{a}} \pm 0.06$ & $17^{\mathrm{b}} \pm 0.91$ & $5^{\mathrm{c}} \pm 1.47$ & $25^{\mathrm{b}} \pm 1.43$ \\
\hline $50 \times 20 \mathrm{~cm}$ & $4.33^{\mathrm{b}} \pm 0.19$ & $5.98^{\mathrm{b}} \pm 0.20$ & $4.56^{\mathrm{b}} \pm 0.14$ & $1.52^{\mathrm{b}} \pm 0.08$ & $0.41^{\mathrm{b}} \pm 0.09$ & $1.65^{\mathrm{a}} \pm 0.09$ & $38^{\mathrm{a}} \pm 1.70$ & $7^{\mathrm{b}} \pm 2.41$ & $44^{\mathrm{a}} \pm 2.33$ \\
\hline $50 \times 30 \mathrm{~cm}$ & $3.07^{\mathrm{c}} \pm 0.12$ & $3.65^{\mathrm{c}} \pm 0.17$ & $3.51^{\mathrm{c}} \pm 0.24$ & $1.96^{\mathrm{a}} \pm 0.08$ & $0.35^{\mathrm{b}} \pm 0.09$ & $1.49^{\mathrm{a}} \pm 0.14$ & $37^{\mathrm{a}} \pm 2.35$ & $9^{\mathrm{a}} \pm 2.05$ & $52^{\mathrm{a}} \pm 2.90$ \\
\hline \multicolumn{10}{|c|}{ Nitrogen } \\
\hline $\mathrm{N}_{0}$ & $4.33^{\mathrm{c}} \pm 0.41$ & $5.23^{\mathrm{c}} \pm 0.45$ & $4.91^{\mathrm{a}} \pm 0.44$ & $1.45^{\mathrm{a}} \pm 0.11$ & $0.31^{c} \pm 0.05$ & $1.20^{\mathrm{b}} \pm 0.21$ & $44^{\mathrm{a}} \pm 4.62$ & $7^{\mathrm{a}} \pm 0.65$ & $33^{\mathrm{b}} \pm 3.84$ \\
\hline $\mathrm{N}_{30}$ & $5.10^{\mathrm{b}} \pm 0.45$ & $5.85^{\mathrm{b}} \pm 0.38$ & $4.92^{\mathrm{a}} \pm 0.58$ & $1.55^{\mathrm{a}} \pm 0.04$ & $0.39^{\mathrm{bc}} \pm 0.12$ & $1.34^{\mathrm{b}} \pm 0.12$ & $43^{\mathrm{a}} \pm 0.78$ & $7^{\mathrm{a}} \pm 1.99$ & $35^{\mathrm{ab}} \pm 3.76$ \\
\hline $\mathrm{N}_{60}$ & $5.84^{\mathrm{a}} \pm 0.47$ & $5.96^{\mathrm{ab}} \pm 0.53$ & $5.62^{\mathrm{a}} \pm 0.60$ & $1.59^{\mathrm{a}} \pm 0.11$ & $0.44^{\mathrm{b}} \pm 0.11$ & $2.00^{\mathrm{a}} \pm 0.08$ & $36^{\mathrm{b}} \pm 2.51$ & $7^{\mathrm{a}} \pm 3.63$ & $50^{\mathrm{a}} \pm 0.69$ \\
\hline $\mathrm{N}_{90}$ & $5.52^{\mathrm{ab}} \pm 0.48$ & $6.46^{\mathrm{a}} \pm 0.59$ & $5.33^{\mathrm{a}} \pm 0.58$ & $1.58^{\mathrm{a}} \pm 0.15$ & $0.52^{\mathrm{a}} \pm 0.08$ & $1.63^{\mathrm{ab}} \pm 0.21$ & $37^{\mathrm{b}} \pm 4.96$ & $7^{\mathrm{a}} \pm 0.82$ & $41^{\mathrm{ab}} \pm 3.40$ \\
\hline
\end{tabular}

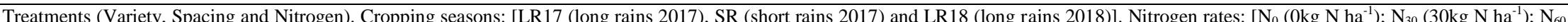
$\left(60 \mathrm{~kg} \mathrm{~N} \mathrm{ha}^{-1}\right)$ and $\mathrm{N}_{90}\left(90 \mathrm{~kg} \mathrm{~N} \mathrm{ha}^{-1}\right)$ ]. Sources of interactions: Variety (V), Spacing (S), N (Nitrogen), VS (Variety x Spacing), VN (Variety x Nitrogen), SN (Spacing x Nitrogen) and VSN (Variety $x$ Spacing $x$ Nitrogen). Means with same letter in a column are not significantly different at $\mathrm{P} \leq 0.05$; Ns means not significant at $\mathrm{P} \leq 0.05$. 
$(\mathrm{P}<0.05)$ affected under LR2017 and LR2018 growth conditions by nitrogen application. Application of $\mathrm{N}_{0}$ and $\mathrm{N}_{30}$ generated the highest harvest index of $44 \%$ and $43 \%$ in LR2017 respectively while for the LR2018, $\mathrm{N}_{60}$ gave the highest harvest index of $50 \%$ but this was statistically similar to $\mathrm{N}_{30}$ and $\mathrm{N}_{90}$ (Table 2).

\subsection{Interactive effects between treatments}

\subsubsection{Synergetic effects of varieties and spacing on plant height and yield indicators}

The effects of interactions between chickpea varieties and spacing on plant height and yield indicators are as shown in Table 3. The highest plant height was recorded for Mwanza 2 in all spacing compared to other varieties; however, Chaina Ix $50 \times 10 \mathrm{~cm}$ spacing posted the highest plant height of $34.8 \mathrm{~cm}$ at H56DAS. Further, Chaina I and Chaina IIIx $50 \times 20 \mathrm{~cm}$ gave higher heights at 56 DAS as compared to other spacing (Table 3). All varieties expressed the highest biomass under spacing $50 \times 10 \mathrm{~cm}$ (Table 3). Further, Mwanza 2x50x10cm spacing gave the highest biomass of $10.6 \mathrm{t} \mathrm{ha}^{-1}$ compared to the lowest Chaina I that had $2.78 \mathrm{tha}^{-1}$. Further, this treatment gave the highest grain yield $\left(1.67 \mathrm{t} \mathrm{ha}^{-1}\right)$ at $50 \times 30 \mathrm{~cm}$ over the cropping period. Saina $\mathrm{K}$ and Chaina III performed better under $50 \times 20 \mathrm{~cm}$ spacing while Mwanza 2 and Chaina I were found better than others at the $50 \times 30 \mathrm{~cm}$ spacing (Table 3 ). Among the all tested varieties, the highest harvest index was reported at $50 \times 30 \mathrm{~cm}$ growth conditions.

\subsubsection{Interactive effects between varieties and nitrogen rates on height and yield attributes}

Mwanza $2 \mathrm{xN}_{90}$ gave the overall highest plant height at 56, 70 and 84 DAS compared to the other varieties and N-rates. However, each variety improved height at different levels of nitrogen, among the tested varieties, Saina $\mathrm{K}$ had high heights at $\mathrm{N}_{30}$, Chaina I at $\mathrm{N}_{90}$, while Chaina III gave the highest value at $\mathrm{N}_{0}$ (Table 4). The $\mathrm{N}_{0}$ treatment resulted in the lowest biomass in all varieties while $\mathrm{N}_{90}$ gave the highest biomass in Mwanza $2\left(7.74 \mathrm{t} \mathrm{ha}^{-1}\right)$. Additionally, Saina $\mathrm{K}$ and Chaina I gave their maximum biomass at $\mathrm{N}_{60}$, while Chaina III was maximum at $\mathrm{N}_{30}$.

Saina $\mathrm{K}$ recorded the highest overall grain yield under $\mathrm{N}_{60}(1.89 \mathrm{t}$ $\mathrm{ha}^{-1}$ ) while the worst performer was Chaina III under $\mathrm{N}_{90}$. However, Mwanza 2 gave maximum yields at $\mathrm{N}_{90}\left(1.73 \mathrm{t} \mathrm{ha}^{-1}\right)$, Chaina I, and Chaina III at $\mathrm{N}_{60}$. Kabuli varieties (Mwanza 2 and Saina K) performed poorly under low N, while Desi varieties (Chaina I and Chaina III) performed lowest under high nitrogen rates. The highest harvest index was reported for the Chaina I (37\%) under $\mathrm{N}_{0}$ while the lowest index was reported for the Chaina III under $\mathrm{N}_{90}$. Saina $\mathrm{K}$ and Mwanza 2 expressed their maximum index under $\mathrm{N}_{60}$, and $\mathrm{N}_{90}$ respectively (Table 4).

\subsubsection{Interactive effects of varieties, spacing and nitrogen rates} on plant height and yield parameters

Mwanza 2 and Chaina I accumulatively performed better at $50 \times 10 \mathrm{cmxN}_{90}$ compared to other varieties (Table 5). At a spacing of $50 \times 20 \mathrm{cmxN}_{60}$, Chaina III gave better performance and gave $36.2 \mathrm{~cm}$ height which is superior to the rest combinations. At $50 \times 30 \mathrm{cmxN}_{0}$, plant height was better among all varieties except in Chaina I which performed better at $50 \times 30 \mathrm{cmxN}_{30}$ (Table 5). Further, Mwanza 2 gave a better plant height of $36.7 \mathrm{~cm}$ at $50 \times 30 \mathrm{~cm} \mathrm{~N}_{90}$ compared to other varieties.

In all interactions involving $\mathrm{N}_{0}$ for biomass production, Mwanza 2 was better than other varieties apart from under $50 \times 30 \mathrm{cmxN}_{0}$ where Saina $\mathrm{K}$ was better (Table 5). Interactions of varieties under $\mathrm{N}_{30}$ fertilization rate resulted in Mwanza giving the highest biomass at $50 \times 10 \mathrm{~cm}, 50 \times 30 \mathrm{~cm}$ except under $50 \times 20 \mathrm{~cm}$ where Saina $\mathrm{K}$ was superior. Additionally, Mwanza 2 gave better biomass under interactions of $\mathrm{N}_{60}$ and $\mathrm{N}_{90}$ except under $50 \times 30 \mathrm{~cm}$ where Saina $\mathrm{K}$ was a better yielder.

In all interactions where the $\mathrm{N}_{0}$ rate was applied, Mwanza 2 performed better in grain yield than all varieties except under $50 \times 30 \mathrm{cmxN}_{0}$ where Chaina I marginally gave the highest results (Table 5). Mwanza 2 performed better where $\mathrm{N}_{90}$ interactions were used except under $50 \times 20 \mathrm{cmxN}_{90}$ where Saina $\mathrm{K}$ recorded the highest grain yield. In addition, Saina $\mathrm{K}$ performed better than other varieties at interactions of $50 x 10 \mathrm{cmxN}_{60}$, and $50 \times 20 \mathrm{cmxN}_{60}$ (Table 5). Additionally, Chaina III gave the highest grain yield with interactions of $50 \mathrm{x} 10 \mathrm{cmxN}_{30}$ of $1.38 \mathrm{t}$ $\mathrm{ha}^{-1}$ compared to other varieties, while Mwanza 2 gave the highest grain yield under $50 \times 20 \mathrm{~cm} \mathrm{x} \mathrm{N}_{30}$ and $50 \times 30 \mathrm{~cm} \mathrm{x} \mathrm{N}_{30}$ of 1.37 and $1.76 \mathrm{t}$ $\mathrm{ha}^{-1}$ respectively.

Significant harvest index among interactions occurred under Saina $\mathrm{K}$ and Chaina I with $50 \times 10 \mathrm{cmxN}_{0}$, and $50 \times 10 \mathrm{cmxN}_{90}$ giving higher results of $20 \%$ compared to Mwanza 2 and Chaina III (Table 5). Interactions of $50 \mathrm{x} 10 \mathrm{cmxN}_{30}$ gave the lowest harvest index for Chaina I but higher results in the other varieties, while in the case of $50 \times 10 \mathrm{~cm} \times \mathrm{N}_{60}$ lowest harvest index was reported for Mwanza 2. Additionally, interactions of Chaina I with $50 \times 30 \mathrm{~cm} \mathrm{~N}_{0}$ gave a better harvest index of $60 \%$ compared to other varieties. Saina K, Mwanza 2, and Chaina III reported a high harvest index under $\mathrm{N}_{30} \times 50 \times 10 \mathrm{~cm}$ and $\mathrm{N}_{30} \times 50 \times 20 \mathrm{~cm}$ while Chaina I was highest under $\mathrm{N}_{30} \times 50 \times 30 \mathrm{~cm}$. Nonetheless, Saina K, Chaina I, and Chaina III gave a higher harvest index with interactions $50 \mathrm{x} 10 \mathrm{cmxN}_{60}$ compared to Mwanza 2, while Saina $\mathrm{K}$ gave the highest index of $30 \%$ under $50 \times 20 \mathrm{cmxN}_{60}$. However, all varieties expressed a better harvest index under $50 \times 30 \mathrm{cmxN}_{60}$, with Mwanza 2 giving a superior index of $60 \%$. Saina $\mathrm{K}$ and Chaina I gave a higher harvest index of $20 \%$ under $50 \times 10 \mathrm{cmxN}_{90}$, while Saina K, Mwanza 2, and Chaina I were better under $50 \times 20 \mathrm{cmxN}_{90}$. Mwanza 2 had a superior harvest index of $50 \%$ under $50 \times 30 \mathrm{cmxN}_{90}$ interactions. 
Effects of Variety, Spacing and Nitrogen Application on Chickpea in Embu County, Kenya

Table 3 Synergetic interactions between variety and spacing on plant height, total biomass, grain yield and harvest index

\begin{tabular}{|c|c|c|c|c|c|c|c|c|c|c|c|c|}
\hline Treatments & SainaK & Mwanza2 & ChainaI & ChainaIII & SainaK & Mwanza2 & ChainaI & ChainaIII & SainaK & Mwanza2 & ChainaI & ChainaIII \\
\hline & \multicolumn{4}{|c|}{ H56 } & \multicolumn{4}{|c|}{$\mathrm{H} 70$} & \multicolumn{4}{|c|}{ H84 } \\
\hline $50 \times 10 \mathrm{~cm}$ & $34.0^{\mathrm{b}} \pm 0.47$ & $34.7^{\mathrm{a}} \pm 0.38$ & $34.8^{\mathrm{a}} \pm 0.38$ & $34.5^{\mathrm{b}} \pm 0.38$ & $35.1^{\mathrm{b}} \pm 0.40$ & $36.3^{\mathrm{a}} \pm 0.41$ & $35.4^{\mathrm{b}} \pm 0.39$ & $34.6^{c} \pm 0.41$ & $36.0^{\mathrm{b}} \pm 0.33$ & $36.8^{\mathrm{a}} \pm 0.39$ & $35.5^{\mathrm{c}} \pm 0.40$ & $34.3^{\mathrm{d}} \pm 0.42$ \\
\hline $50 \times 20 \mathrm{~cm}$ & $33.1^{b} \pm 0.52$ & $33.2^{b} \pm 0.58$ & $34.4^{a} \pm 0.31$ & $34.2^{\mathrm{a}} \pm 0.53$ & $34.4^{\mathrm{d}} \pm 0.44$ & $36.4^{\mathrm{a}} \pm 0.46$ & $35.6^{\mathrm{b}} \pm 0.36$ & $34.9^{c} \pm 0.39$ & $36.5^{b} \pm 0.39$ & $37.5^{\mathrm{a}} \pm 0.39$ & $35.7^{c} \pm 0.38$ & $35.4^{\mathrm{c}} \pm 0.43$ \\
\hline $50 \times 30 \mathrm{~cm}$ & $33.0^{\mathrm{c}} \pm 0.52$ & $35.3^{\mathrm{a}} \pm 0.58$ & $34.0^{b} \pm 0.37$ & $35.1^{\mathrm{a}} \pm 0.35$ & $34.3^{\mathrm{c}} \pm 0.42$ & $36.8^{\mathrm{a}} \pm 0.40$ & $35.9^{\mathrm{b}} \pm 0.45$ & $36.0^{\mathrm{b}} \pm 0.46$ & $36.3^{\mathrm{b}} \pm 0.35$ & $39.2^{\mathrm{a}} \pm 0.52$ & $36.3^{\mathrm{b}} \pm 0.38$ & $36.3^{b} \pm 0.41$ \\
\hline \multicolumn{13}{|c|}{ Yield attributes } \\
\hline & \multicolumn{4}{|c|}{$\mathrm{Tb}\left(\right.$ tha $\left.^{-1}\right)$} & \multicolumn{4}{|c|}{ GY $\left(\right.$ tha $\left.^{-1}\right)$} & \multicolumn{4}{|c|}{$\mathrm{HI} \%$} \\
\hline $50 \times 10 \mathrm{~cm}$ & $9.38^{\mathrm{b}} \pm 0.56$ & $10.6^{\mathrm{a}} \pm 0.70$ & $5.63^{\mathrm{c}} \pm 0.41$ & $5.98^{\mathrm{c}} \pm 0.37$ & $1.21^{\mathrm{a}} \pm 0.09$ & $1.16^{\mathrm{a}} \pm 0.14$ & $0.89^{\mathrm{a}} \pm 0.14$ & $0.92^{\mathrm{a}} \pm 0.16$ & $17.2^{\mathrm{c}} \pm 0.01$ & $12.5^{\mathrm{c}} \pm 0.02$ & $16.1^{\mathrm{c}} \pm 0.02$ & $16.3^{c} \pm 0.02$ \\
\hline $50 \times 20 \mathrm{~cm}$ & $6.32^{\mathrm{a}} \pm 0.56$ & $6.56^{\mathrm{a}} \pm 0.41$ & $3.42^{b} \pm 0.25$ & $3.52^{\mathrm{b}} \pm 0.33$ & $1.64^{\mathrm{a}} \pm 0.27$ & $1.51^{\mathrm{a}} \pm 0.16$ & $0.86^{\mathrm{b}} \pm 0.09$ & $0.76^{\mathrm{bc}} \pm 0.07$ & $32.1^{\mathrm{b}} \pm 0.05$ & $29.7^{\mathrm{b}} \pm 0.03$ & $28.9^{\mathrm{b}} \pm 0.04$ & $27.2^{b} \pm 0.04$ \\
\hline $50 \times 30 \mathrm{~cm}$ & $4.07^{\mathrm{a}} \pm 0.22$ & $3.99^{\mathrm{a}} \pm 0.28$ & $2.78^{\mathrm{b}} \pm 0.32$ & $2.79^{\mathrm{b}} \pm 0.33$ & $1.43^{\mathrm{a}} \pm 0.17$ & $1.67^{\mathrm{a}} \pm 0.15$ & $1.17^{\mathrm{b}} \pm 0.16$ & $0.79^{\mathrm{bc}} \pm 0.21$ & $40.9^{\mathrm{a}} \pm 0.05$ & $50.0^{\mathrm{a}} \pm 0.03$ & $43.4^{\mathrm{a}} \pm 0.05$ & $34.4^{\mathrm{a}} \pm 0.09$ \\
\hline
\end{tabular}

Variety (Saina K, Mwanza 2, Chaina I and Chaina III). H56 means 56 days after sowing; H70 means 70 days after sowing; H84 means 84 days after sowing. N-rates: $\mathrm{N}_{0}\left(0 \mathrm{~kg} \mathrm{~N}\right.$ ha $\left.{ }^{-1}\right), \mathrm{N}_{30}\left(30\right.$ kg N ha $\left.{ }^{-1}\right), \mathrm{N}_{60}$

$\left(60 \mathrm{~kg} \mathrm{~N} \mathrm{ha}^{-1}\right)$ and $\mathrm{N}_{90}\left(90 \mathrm{~kg} \mathrm{~N} \mathrm{ha}^{-1}\right)$. Tb means Total biomass; GY means economic grain yield; tha ${ }^{-1}$ means tonnes per hectare; HI means harvest index.

Table 4 Synergetic interactions between variety and nitrogen on plant height and yield attributes

\begin{tabular}{|c|c|c|c|c|c|c|c|c|c|c|c|c|}
\hline $\begin{array}{c}\text { Varietyx } \\
\text { N }\end{array}$ & SainaK & Mwanza2 & ChainaI & ChainaIII & SainaK & Mwanza2 & ChainaI & ChainaIII & SainaK & Mwanza 2 & ChainaI & ChainaIII \\
\hline & \multicolumn{4}{|c|}{ H56 } & \multicolumn{4}{|c|}{$\mathrm{H} 70$} & \multicolumn{4}{|c|}{ H84 } \\
\hline $\mathrm{N}_{0}$ & $33.0^{\mathrm{b}} \pm 1.21$ & $33.3^{\mathrm{b}} \pm 1.24$ & $34.6^{\mathrm{a}} \pm 0.85$ & $34.5^{\mathrm{a}} \pm 1.07$ & $35.0^{\mathrm{b}} \pm 0.66$ & $35.7^{\mathrm{a}} \pm 0.66$ & $35.3 \mathrm{a} \pm 0.74$ & $34.6 \mathrm{~b} \pm 0.91$ & $36.3^{\mathrm{a}} \pm 0.73$ & $36.3^{\mathrm{a}} \pm 0.99$ & $35.2^{\mathrm{b}} \pm 0.87$ & $35.9^{\mathrm{a}} \pm 1.17$ \\
\hline $\mathrm{N}_{30}$ & $33.7^{\mathrm{b}} \pm 0.76$ & $33.0^{\mathrm{c}} \pm 1.64$ & $35.8^{\mathrm{a}} \pm 0.81$ & $33.9^{\mathrm{b}} \pm 0.66$ & $34.5^{\mathrm{c}} \pm 0.52$ & $36.2^{\mathrm{a}} \pm 0.71$ & $35.7^{\mathrm{b}} \pm 0.51$ & $34.6^{\mathrm{c}} \pm 1.03$ & $36.6^{\mathrm{a}} \pm 0.57$ & $37.0^{\mathrm{a}} \pm 1.06$ & $36.2^{\mathrm{b}} \pm 1.00$ & $34.6^{\mathrm{c}} \pm 1.45$ \\
\hline $\mathrm{N}_{60}$ & $33.9^{c} \pm 1.58$ & $34.4^{\mathrm{b}} \pm 0.98$ & $35.6^{\mathrm{a}} \pm 0.88$ & $35.7^{\mathrm{a}} \pm 0.81$ & $34.1^{\mathrm{c}} \pm 1.03$ & $36.4^{\mathrm{a}} \pm 0.74$ & $35.5^{\mathrm{b}} \pm 0.59$ & $35.8^{\mathrm{b}} \pm 0.42$ & $35.8^{\mathrm{b}} \pm 1.12$ & $38.5^{\mathrm{a}} \pm 1.68$ & $35.7^{\mathrm{b}} \pm 1.09$ & $35.7^{\mathrm{b}} \pm 0.98$ \\
\hline $\mathrm{N}_{90}$ & $34.2^{\mathrm{b}} \pm 0.43$ & $36.1^{\mathrm{a}} \pm 1.39$ & $35.6^{\mathrm{a}} \pm 0.57$ & $34.7^{\mathrm{b}} \pm 0.69$ & $34.9^{\mathrm{d}} \pm 0.61$ & $37.7^{\mathrm{a}} \pm 0.85$ & $36.1^{\mathrm{b}} \pm 0.71$ & $35.6^{\mathrm{c}} \pm 0.57$ & $36.4^{\mathrm{b}} \pm 0.94$ & $39.5^{\mathrm{a}} \pm 1.49$ & $36.3^{\mathrm{b}} \pm 0.78$ & $35.2^{\mathrm{c}} \pm 1.13$ \\
\hline \multicolumn{13}{|c|}{ Yield attributes } \\
\hline & \multicolumn{4}{|c|}{$\mathrm{Tb}\left(\right.$ tha $\left.^{-1}\right)$} & \multicolumn{4}{|c|}{ GY $\left(\right.$ tha $\left.^{-1}\right)$} & \multicolumn{4}{|c|}{$\mathrm{HI} \%$} \\
\hline $\mathrm{N}_{0}$ & $5.5^{\mathrm{b}} \pm 0.77$ & $6.9^{\mathrm{a}} \pm 1.11$ & $3.1 \mathrm{~d} \pm 0.37$ & $3.8^{\mathrm{c}} \pm 0.42$ & $1.1^{\mathrm{a}} \pm 0.09$ & $1.2^{\mathrm{a}} \pm 0.23$ & $0.9^{\mathrm{a}} \pm 0.14$ & $0.7^{\mathrm{a}} \pm 0.08$ & $26.5^{\mathrm{b}} \pm 0.04$ & $26.3^{\mathrm{b}} \pm 0.06$ & $36.6^{\mathrm{a}} \pm 0.06$ & $23.3^{\mathrm{c}} \pm 0.04$ \\
\hline $\mathrm{N}_{30}$ & $6.3^{\mathrm{a}} \pm 0.92$ & $6.2^{\mathrm{a}} \pm 0.79$ & $4.2^{\mathrm{b}} \pm 0.56$ & $4.5^{\mathrm{b}} \pm 0.68$ & $1.1^{\mathrm{a}} \pm 0.13$ & $1.4^{\mathrm{a}} \pm 0.20$ & $1.0^{\mathrm{a}} \pm 0.19$ & $1.0^{\mathrm{a}} \pm 0.18$ & $25.0^{c} \pm 0.04$ & $30.9^{\mathrm{a}} \pm 0.05$ & $28.3^{\mathrm{b}} \pm 0.06$ & $27.8^{\mathrm{b}} \pm 0.05$ \\
\hline $\mathrm{N}_{60}$ & $7.39^{\mathrm{a}} \pm 0.90$ & $7.27^{\mathrm{a}} \pm 1.126$ & $4.49^{\mathrm{b}} \pm 0.68$ & $4.08^{\mathrm{b}} \pm 0.73$ & $1.89^{\mathrm{a}} \pm 0.29$ & $1.4^{\mathrm{a}} \pm 0.21$ & $1.0^{\mathrm{b}} \pm 0.15$ & $1.0^{\mathrm{b}} \pm 0.31$ & $36.0 \mathrm{a} \pm 0.07$ & $29.9 c \pm 0.07$ & $26.7 \mathrm{~d} \pm 0.05$ & $32.0 \mathrm{~b} \pm 0.11$ \\
\hline $\mathrm{N}_{90}$ & $7.2^{\mathrm{b}} \pm 1.11$ & $7.8^{\mathrm{a}} \pm 1.37$ & $4.0^{\mathrm{b}} \pm 0.61$ & $4.0^{\mathrm{b}} \pm 0.55$ & $1.7^{\mathrm{a}} \pm 0.3$ & $1.7^{\mathrm{a}} \pm 0.23$ & $1.0^{\mathrm{b}} \pm 0.16$ & $0.6^{\mathrm{b}} \pm 0.07$ & $32.7^{b} \pm 0.06$ & $34.1^{\mathrm{a}} \pm 0.06$ & $26.3^{\mathrm{c}} \pm 0.04$ & $20.8^{\mathrm{d}} \pm 0.04$ \\
\hline
\end{tabular}

Variety (Saina K, Mwanza 2, Chaina I and Chaina III). H56 means 56 days after sowing; H70 means 70 days after sowing; H84 means 84 days after sowing. N-rates: $\mathrm{N}_{0}\left(0 \mathrm{~kg} \mathrm{~N}\right.$ ha $\left.{ }^{-1}\right), \mathrm{N}_{30}\left(30\right.$ kg N ha $\left.{ }^{-1}\right)$, $\mathrm{N}_{60}$ $\left(60 \mathrm{~kg} \mathrm{~N} \mathrm{ha}^{-1}\right)$ and $\mathrm{N}_{90}\left(90 \mathrm{~kg} \mathrm{~N} \mathrm{ha}^{-1}\right)$. Tb means Total biomass; GY means economic grain yield; tha ${ }^{-1}$ means tonnes per hectare; HI means harvest index.

Journal of Experimental Biology and Agricultural Sciences

http://www.jebas.org 
Table 5 Effect of synergetic interactions between varieties, spacing and nitrogen rates on plant height and yield attributes

\begin{tabular}{|c|c|c|c|c|c|c|c|c|c|c|c|c|c|c|c|c|}
\hline & K1 & K2 & D1 & D2 & K1 & K2 & D1 & D2 & K1 & $\mathrm{K} 2$ & D1 & D2 & K1 & $\mathrm{K} 2$ & D1 & D2 \\
\hline Treatments & \multicolumn{4}{|c|}{$\mathrm{H} 84 \mathrm{~cm}$} & \multicolumn{4}{|c|}{$\mathrm{Tb}\left(\mathrm{tha}^{-1}\right)$} & \multicolumn{4}{|c|}{ GY $\left(t h a^{-1}\right)$} & \multicolumn{4}{|c|}{$\mathrm{HI} \%$} \\
\hline $50 \times 10 \mathrm{cmxN}_{0}$ & $33.5^{\mathrm{b}} \pm 0.31$ & $34.1^{\mathrm{a}} \pm 0.53$ & $34.3^{\mathrm{a}} \pm 0.67$ & $34.0^{\mathrm{a}} \pm 0.92$ & $5.8^{\mathrm{b}} \pm 0.14$ & $10.7^{\mathrm{a}} \pm 0.3$ & $4.7^{\mathrm{c}} \pm 0.57$ & $5.1^{\mathrm{c}} \pm 0.34$ & $0.9^{\mathrm{a}} \pm 0.07$ & $1.1^{\mathrm{a}} \pm 0.18$ & $0.6^{\mathrm{b}} \pm 0.06$ & $0.6^{\mathrm{b}} \pm 0.04$ & $20^{\mathrm{a}} \pm 0.02$ & $10^{\mathrm{a}} \pm 0.02$ & $20^{\mathrm{a}} \pm 0.01$ & $10^{\mathrm{a}} \pm 0.02$ \\
\hline $50 \times 10 \mathrm{cmxN}_{30}$ & $33.1^{\mathrm{c}} \pm 0.49$ & $33.5^{\mathrm{c}} \pm 0.74$ & $35.8^{\mathrm{a}} \pm 0.87$ & $34.7^{b} \pm 0.74$ & $7.6^{\mathrm{a}} \pm 0.35$ & $8.0^{\mathrm{a}} \pm 1.75$ & $6.7^{b} \pm 0.20$ & $6.8^{\mathrm{b}} \pm 0.41$ & $1.1^{\mathrm{a}} \pm 0.16$ & $1.1^{\mathrm{a}} \pm 0.13$ & $0.8^{\mathrm{a}} \pm 0.09$ & $1.4^{\mathrm{a}} \pm 0.39$ & $20^{\mathrm{a}} \pm 0.02$ & $20^{\mathrm{a}} \pm 0.03$ & $10^{\mathrm{b}} \pm 0.02$ & $20^{\mathrm{a}} \pm 0.05$ \\
\hline $50 \times 10 \mathrm{cmxN}_{60}$ & $33.2^{\mathrm{c}} \pm 0.73$ & $34.9^{\mathrm{b}} \pm 0.89$ & $35.7^{\mathrm{a}} \pm 1.26$ & $35.7^{\mathrm{a}} \pm 1.07$ & $8.4^{\mathrm{b}} \pm 1.01$ & $10.4^{\mathrm{a}} \pm 0.13$ & $7.1^{\mathrm{c}} \pm 0.64$ & $6.8^{c} \pm 0.75$ & $1.5^{\mathrm{a}} \pm 0.13$ & $0.9^{\mathrm{c}} \pm 0.05$ & $1.3^{\mathrm{a}} \pm 0.15$ & $1.2^{\mathrm{b}} \pm 0.09$ & $20^{\mathrm{a}} \pm 0.02$ & $10^{\mathrm{b}} \pm 0.01$ & $20^{\mathrm{a}} \pm 0.03$ & $20^{\mathrm{a}} \pm 0.01$ \\
\hline $50 \times 10 \mathrm{cmxN}_{90}$ & $33.8^{\mathrm{c}} \pm 0.75$ & $36.6^{\mathrm{a}} \pm 0.66$ & $36.0^{\mathrm{b}} \pm 0.52$ & $33.8^{\mathrm{c}} \pm 0.46$ & $8.4^{\mathrm{b}} \pm 0.99$ & $14^{\mathrm{a}} \pm 0.98$ & $5.5^{\mathrm{d}} \pm 1.18$ & $6.8^{\mathrm{c}} \pm 0.38$ & $1.4^{\mathrm{a}} \pm 0.13$ & $1.6^{\mathrm{a}} \pm 0.22$ & $0.9^{\mathrm{b}} \pm 0.44$ & $0.6^{\mathrm{bc}} \pm 0.05$ & $20^{\mathrm{a}} \pm 0.04$ & $10^{\mathrm{b}} \pm 0.02$ & $2^{\mathrm{a}} \pm 0.07$ & $10^{\mathrm{b}} \pm 0.01$ \\
\hline $50 \times 20 \mathrm{cmxN}_{0}$ & $31.8^{\mathrm{c}} \pm 0.38$ & $31.5^{\mathrm{c}} \pm 1.08$ & $34.2^{\mathrm{a}} \pm 0.56$ & $33.3^{\mathrm{b}} \pm 0.95$ & $6.3^{\mathrm{b}} \pm 1.01$ & $8.8^{\mathrm{a}} \pm 0.38$ & $2.6^{\mathrm{d}} \pm 0.22$ & $5.1^{\mathrm{c}} \pm 0.34$ & $1.1^{\mathrm{a}} \pm 0.09$ & $1.2^{\mathrm{a}} \pm 0.34$ & $0.9^{\mathrm{a}} \pm 0.08$ & $0.9^{\mathrm{a}} \pm 0.06$ & $30^{\mathrm{b}} \pm 0.03$ & $20^{\mathrm{c}} \pm 0.05$ & $40^{\mathrm{a}} \pm 0.02$ & $20^{\mathrm{a}} \pm 0.04$ \\
\hline $50 \times 20 \mathrm{cmxN}_{30}$ & $33.5^{\mathrm{b}} \pm 0.72$ & $33.6^{\mathrm{b}} \pm 0.46$ & $35.1^{\mathrm{a}} \pm 1.05$ & $33.0^{c} \pm 0.89$ & $6.7^{\mathrm{a}} \pm 0.17$ & $4.9^{\mathrm{b}} \pm 0.33$ & $3.7^{\mathrm{d}} \pm 0.20$ & $4.1^{\mathrm{c}} \pm 0.31$ & $1.1^{\mathrm{a}} \pm 0.15$ & $1.4^{\mathrm{a}} \pm 0.20$ & $0.8^{\mathrm{b}} \pm 0.16$ & $0.8^{\mathrm{b}} \pm 0.08$ & $30^{\mathrm{a}} \pm 0.03$ & $30^{\mathrm{a}} \pm 0.03$ & $20^{\mathrm{b}} \pm 0.05$ & $30^{\mathrm{a}} \pm 0.04$ \\
\hline $50 \times 20 \mathrm{cmxN}_{60}$ & $31.6^{\mathrm{d}} \pm 0.81$ & $34.1^{\mathrm{c}} \pm 1.05$ & $35.4^{\mathrm{b}} \pm 0.78$ & $36.2^{\mathrm{a}} \pm 0.59$ & $7.2^{\mathrm{a}} \pm 1.00$ & $7.3^{\mathrm{a}} \pm 0.80$ & $2.4^{b} \pm 0.34$ & $2.1^{\mathrm{b}} \pm 0.13$ & $2.1^{\mathrm{a}} \pm 0.50$ & $1.4^{\mathrm{b}} \pm 0.17$ & $0.7^{\mathrm{c}} \pm 0.16$ & $0.5^{\mathrm{c}} \pm 0.07$ & $30^{\mathrm{a}} \pm 0.06$ & $20^{\mathrm{b}} \pm 0.04$ & $20^{\mathrm{b}} \pm 0.04$ & $20^{\mathrm{b}} \pm 0.04$ \\
\hline $50 \times 20 \mathrm{cmxN}_{90}$ & $31.3^{\mathrm{b}} \pm 0.34$ & $35.0^{\mathrm{a}} \pm 0.48$ & $35.5^{\mathrm{a}} \pm 0.90$ & $35.2^{\mathrm{a}} \pm 0.64$ & $5.9^{\mathrm{b}} \pm 1.25$ & $7.2^{\mathrm{a}} \pm 0.46$ & $2.7^{\mathrm{d}} \pm 0.53$ & $3.6^{\mathrm{c}} \pm 0.44$ & $2.3^{\mathrm{a}} \pm 0.65$ & $2.2^{\mathrm{a}} \pm 0.13$ & $1.1^{\mathrm{b}} \pm 0.15$ & $0.8^{\mathrm{b}} \pm 0.15$ & $40^{\mathrm{a}} \pm 0.05$ & $40^{\mathrm{a}} \pm 0.03$ & $40^{\mathrm{a}} \pm 0.04$ & $30^{\mathrm{b}} \pm 0.07$ \\
\hline $50 \times 30 \mathrm{cmxN}_{0}$ & $33.9^{\mathrm{c}} \pm 0.71$ & $34.3^{\mathrm{c}} \pm 0.76$ & $35.2^{\mathrm{b}} \pm 0.79$ & $36.2^{\mathrm{a}} \pm 0.94$ & $3.5^{\mathrm{a}} \pm 0.38$ & $3.1^{\mathrm{a}} \pm 0.23$ & $2.0^{c} \pm 0.26$ & $2.5^{\mathrm{b}} \pm 0.22$ & $1.2^{\mathrm{a}} \pm 0.11$ & $1.4^{\mathrm{a}} \pm 0.11$ & $1.4^{\mathrm{a}} \pm 0.11$ & $0.7^{\mathrm{b}} \pm 0.09$ & $40^{c} \pm 0.06$ & $50^{\mathrm{b}} \pm 0.04$ & $60^{\mathrm{a}} \pm 0.04$ & $30^{\mathrm{d}} \pm 0.04$ \\
\hline $50 \times 30 \mathrm{cmxN}_{30}$ & $32.8^{\mathrm{c}} \pm 0.53$ & $32.0^{\mathrm{d}} \pm 0.89$ & $36.6^{\mathrm{a}} \pm 0.90$ & $34.0^{\mathrm{b}} \pm 0.82$ & $3.8^{\mathrm{b}} \pm 0.13$ & $5.0^{\mathrm{a}} \pm 0.52$ & $2.0^{\mathrm{c}} \pm 0.23$ & $3.6^{\mathrm{b}} \pm 1.16$ & $1.0^{\mathrm{bc}} \pm 0.06$ & $1.8^{\mathrm{a}} \pm 0.11$ & $1.3^{b} \pm 0.29$ & $0.7^{\mathrm{c}} \pm 0.09$ & $30^{\mathrm{c}} \pm 0.02$ & $40^{\mathrm{b}} \pm 0.03$ & $50^{\mathrm{a}} \pm 0.05$ & $30^{c} \pm 0.06$ \\
\hline $50 \times 30 \mathrm{cmxN}_{60}$ & $33.7^{\mathrm{c}} \pm 0.80$ & $34.1^{c} \pm 1.32$ & $35.8^{\mathrm{a}} \pm 0.60$ & $35.2^{\mathrm{b}} \pm 0.63$ & $4.9^{\mathrm{a}} \pm 0.20$ & $4.4^{b} \pm 0.46$ & $5.2^{\mathrm{a}} \pm 0.85$ & $2.7^{\mathrm{c}} \pm 0.26$ & $2.1^{\mathrm{a}} \pm 0.32$ & $2.1^{\mathrm{a}} \pm 0.07$ & $1.2^{\mathrm{b}} \pm 0.25$ & $1.4^{\mathrm{b}} \pm 0.77$ & $60^{\mathrm{a}} \pm 0.09$ & $60^{\mathrm{a}} \pm 0.04$ & $40^{c} \pm 0.07$ & $50^{\mathrm{b}} \pm 0.33$ \\
\hline $50 \times 30 \mathrm{cmxN}_{90}$ & $32.3^{\mathrm{c}} \pm 0.63$ & $36.7^{\mathrm{a}} \pm 1.03$ & $35.2^{\mathrm{b}} \pm 0.82$ & $35.0^{\mathrm{b}} \pm 1.19$ & $4.8^{\mathrm{a}} \pm 0.18$ & $4.3^{\mathrm{b}} \pm 0.41$ & $3.0^{c} \pm 0.69$ & $2.2^{\mathrm{d} \pm} \pm 0.21$ & $1.4^{\mathrm{a}} \pm 0.14$ & $1.5^{\mathrm{a}} \pm 0.19$ & $0.9^{b} \pm 0.22$ & $0.5^{\mathrm{b}} \pm 0.04$ & $40^{\mathrm{b}} \pm 0.03$ & $50^{\mathrm{a}} \pm 0.06$ & $30^{c} \pm 0.07$ & $30^{c} \pm 0.01$ \\
\hline
\end{tabular}

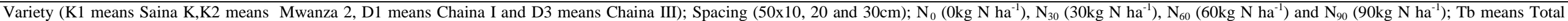
biomass; GY means economic grain yield; tha ${ }^{-1}$ means tonnes per hectare; HI means harvest index 
Table 6 Synergetic effects of season, variety, spacing and nitrogen rates on traits under study

\begin{tabular}{|c|c|c|c|c|c|c|}
\hline Source of Variation & H56 & $\mathrm{H} 70$ & H84 & Total biomass & Grain yield & Harvest index \\
\hline Season & 0.0851 & $0.0006 * * *$ & 0.1582 & $<0.0001 * * *$ & $<0.0001 * * *$ & $<0.0001 * * *$ \\
\hline Season $* \mathrm{~V}$ & $<.0001 * * *$ & 0.0065 & $0.0001 * * *$ & $<0.0001 * * *$ & $<0.0001 * * *$ & $0.0052 *$ \\
\hline Season $* S$ & 0.4149 & $0.0264 * *$ & $<0.0001 * * *$ & $<0.0001 * * *$ & $<0.0001 * * *$ & $<0.0001 * * *$ \\
\hline Season $* V * S$ & $<.0001 * * *$ & $0.0181 * *$ & $0.0089^{*}$ & $<0.0001 * * *$ & $<0.0001 * * *$ & $0.0004 * * *$ \\
\hline Season $* N$ & $<.0001 * * *$ & 0.1872 & $0.0154 * *$ & $0.02 * *$ & $0.003 *$ & $<0.0001 * * *$ \\
\hline Season $* \mathrm{~V} * \mathrm{~N}$ & $<.0001 * * *$ & $0.0537 * *$ & $0.0044 *$ & $<0.0001 * * *$ & $0.03 * *$ & $0.0061^{*}$ \\
\hline Season $* S * N$ & $<.0001 * * *$ & $<.0001 * * *$ & $<0.0001 * * *$ & $0.0001 * * *$ & $<0.0001 * * *$ & $<0.0001 * * *$ \\
\hline Season $* \mathrm{~V} * \mathrm{~S} * \mathrm{~N}$ & $<.0001 * * *$ & $<.0001 * * *$ & $<0.0001^{* * *}$ & $<0.0001 * * *$ & $0.04 * *$ & $0.0045^{*}$ \\
\hline
\end{tabular}

Sources of variation, season, $\mathrm{V}$ indicates variety, $\mathrm{S}$ indicates spacing and $\mathrm{N}$ indicates nitrogen application rates, $\mathrm{P}$-values in bold signify nonsignificance. $* * *$ and $* * *$ means $\mathrm{P}$ is significant at $<.01,<.05$ and $<.001$.

3.3.4 Interactions of season, variety, spacing, and nitrogen application rates on all traits

Interactions between season, variety, spacing, and nitrogen application rates induced a significant effect $(\mathrm{P}<0.05)$ on the various chickpea performance traits (Table 6). Interactions of season $\mathrm{x}$ variety, season $\mathrm{x}$ variety $\mathrm{x}$ nitrogen, Season $\mathrm{x}$ Variety $\mathrm{x}$ Spacing $x$ Nitrogen influenced a significant effect on all studied traits.

\section{Discussions}

In the current study, Mwanza 2 had significantly higher vegetative growth at 70 and 84DAS this was followed by the Chaina I and Chaina III over $7 \%$ at full maturity. The differences in height were realized at the later stages of vegetative growth. Kabuli varieties, under which Mwanza 2 are classified, are taller than Desi varieties (Chaina I and Chaina III) (Onyari et al. 2010; Mallu 2015).

In this study, spacing did not show a clear pattern in various growth stages although variations were observed under different spacing. The tallest plants were observed under $50 \times 30 \mathrm{~cm}$ spacing in all the seasons. These findings corroborate with findings of Agajie (2014) and Under Newly (2011), in chickpea plants and faba beans respectively under wider spacing. Similarly, other works established differences in plant height across different legume crops under varied spacing (Tuarira and Moses 2014; Alemayehu et al. 2015). The utilization of soil nutrients such as added nitrogen is reported to significantly influence chickpea growth, expressed in terms of plant height (Fazle et al. 2014). Though nitrogen is known to promote plant growth, the level varies and depending on the time of nutrient application (Dhima et al. 2015; Caliskan et al. 2008). These results are in agreement with the findings of the current study. At 56 and 70 DAS plant height of the chickpea was conspicuously lower in the $\mathrm{N}_{0}, \mathrm{~N}_{30}$, and $\mathrm{N}_{60}$ compared to the $\mathrm{N}_{90}$ application rates. In later stages of growth (84DAS), significance in plant height between $\mathrm{N}_{90}$ and the other rates $\left(\mathrm{N}_{0}, \mathrm{~N}_{30}\right.$, and $\left.\mathrm{N}_{60}\right)$ reduced, mainly due to senescence (Kherif et al. 2021). The final plant height after $N_{30}$ to $N_{90}$ fertilization gave taller plants indicating that increased $\mathrm{N}$-application rates can positively increase plant growth of chickpea. The increase in plant height under $\mathrm{N}$-fertilized plots is due to readily available Nitrogen from inorganic fertilizer that stimulated crop growth (Namvar et al. 2011; Fazle et al. 2014; Goa and Ashamo 2016).

In the current study, plant biomass reflects the amount of sunlight, water, and mineral resources that a plant can capture and turn into plant mass (Sims et al., 2012; Macák et al. 2020). The spacing of $50 \times 10 \mathrm{~cm}$ had significantly higher biomass compared to the other wider spacing. This was more prominent in Saina K, which produced more than $59 \%$ biomass compared to the other varieties. On the other hand, Mwanza 2, which is a Kabuli, had higher biomass across the seasons with over $78 \%$ compared to the Desi varieties (Chaina I and Chaina III). Differences in biomass production were due to variations in adapting to environmental conditions among varieties (Alemu et al. 2014). Mwanza 2 had a taller stature than Chaina III, reflected in all parts of the plant. This enabled the plants to capture more photosynthetically active radiation (PAR) for increased biomass (Goa 2014; Devi et al. 2019). Results, however, contradict the findings of Mekuanint et al. (2018), who found a non-significant effect of variety in biomass production between two chickpea varieties under three-level spacing with blended fertilizers.

Under $50 \times 10 \mathrm{~cm}$ growth conditions, the biomass of $5.1 \mathrm{tha}^{-1}$ was recorded for chickpea. This was $62 \%$ higher than that of $50 \times 30 \mathrm{~cm}$ spacing. Thus, increased plant density enables chickpeas to produce more biomass (Vaghar et al. 2013). However, the findings contradicted with the findings of Gezahegn et al. (2016), who reported that plant spacing increased biomass in Vicia faba and $P$. vulgaris respectively. $\mathrm{N}$-fertilizer application also increased plant biomass, especially in early growth stages. On average, the $\mathrm{N}_{60}$ and $\mathrm{N}_{90}$ rates had over $20 \%$ more biomass than the control rate $\left(\mathrm{N}_{0}\right)$. 
This corroborates with the findings of Onyari et al. (2010), those who reported 4.6 to $13.6 \mathrm{t} \mathrm{ha}^{-1}$ chickpea biomass in Kenya. Hence, Kabuli varieties with closer spacing and adequate $\mathrm{N}$-fertilizer have the potential of producing higher biomass yield under the Mwea conditions in Kenya. For other legumes, biomass yields of 3.0 and $2.6 \mathrm{t} \mathrm{ha}^{-1}$ have been reported for Bambara nuts when 45 and $0 \mathrm{~kg}-\mathrm{N}$ $\mathrm{ha}^{-1}$ were applied respectively (Uchara et al. 2013).

Crop yield is important in agriculture as it shows the amount of produce harvested per unit area for a given time (Keerthi et al. 2015). Seasons provide different weather conditions for the growth and development of different varieties; hence, they can convert resources to outputs in form of yields (Sellami et al. 2021). In this study, Kabuli (Mwanza 2 and Saina K) varieties gave higher grain yield than Desi type (Chaina I \& Chaina III) grown in three seasons in Mwea, Kenya, indicating that they are better adaptable to the growth conditions. In contradiction Księżak and Bojarszczuk (2020), reported a higher grain yield in Desi than Kabuli under various cropping methods. In addition, Shumi et al. (2020), reported significant differences in grain yields of Chickpea varieties in different seasons.

In the LR2017 season, the amount of rainfall was less than normal and the grain production in the $50 \times 30 \mathrm{~cm}$ spacing performed better than other spacing, which underscored the effect of competition for resources on chickpea, crop production. However, in LR2018 season (rainfall more than normal) had no significant differences in the grain yield among the three plant spacing. This implied that reduced rainfall and wider spacing favored higher grain yield, while higher rainfall promoted vegetative growth at the expense of grain yield (Onyari et al. 2010). Similar observations were reported by Tamiru et al. (2020), who found higher grain yield under wider intra row spacing of $15 \mathrm{~cm}$ in chickpea in drier environments.

Application of $\mathrm{N}_{60}$ gave the highest chickpea grain yields in two seasons compared to lower N-rates, though not significant from $\mathrm{N}_{90}$. However, in all seasons, $\mathrm{N}_{60}$ was significantly higher than $\mathrm{N}_{0}$ and $\mathrm{N}_{30}$ growth conditions in SR2017 and LR2018 seasons. Observation shows that starter nitrogen of about $\mathrm{N}_{60}$ needs to be applied for chickpea production as, low starter nitrogen led to low yields under the conditions of Mwea, Kenya. These results are in agreement with the observation of previous researchers who suggested that the application of nitrogen fertilizers enhances chickpea yield (Uddin et al. 2014). Similarly, Dar et al. (2021), established the highest yield of $2,023 \mathrm{~kg} \mathrm{ha}^{-1}$ under high $\mathrm{N}$ application $\left(30 \mathrm{kgha}^{-1}\right)$ compared to lower rates $\left(0,15 \mathrm{~kg} \mathrm{~N}^{-1}\right)$, and the highest rate of $45 \mathrm{~kg} \mathrm{ha}^{-1} \mathrm{~N}\left(1940 \mathrm{~kg} \mathrm{ha}^{-1}\right)$. In addition, Khaitov and Abdiev (2018), reported high grain yield (1.68 $\left.\mathrm{t} \mathrm{ha}^{-1}\right)$ under $75 \mathrm{kgha}^{-1} \mathrm{~N}$ fertilization compared to lower rates and the highest rate of $100 \mathrm{kgha}^{-1}$ nitrogen. Starter doses of 20 to $40 \mathrm{~kg} \mathrm{~N}$ $\mathrm{ha}^{-1}$ are widely recommended (Dar et al., 2021), but the findings of the current study show that increasing N-rates up to $60 \mathrm{~kg} \mathrm{ha}^{-1}$ can increase the grain yield of chickpea.

The harvest index determines crop potential as it describes the capacity of a crop to allocate biomass into reproductive parts, and is thus used as a measure of reproductive efficiency (Wnuk et al. 2013). The Kabuli varieties had a better harvest index than the Desi varieties. However, various previous researchers reported that the harvest index of Kabuli's is reportedly inferior to that of Desi (Richards et al. 2019; Maya and Maphosa 2020). Mekuanint et al. (2018) reported contradictory results of a high harvest index in Desi (54\%) compared to Kabuli (48\%). Bhardwaj and Hamama (2015) reported similar results in mung bean (Vigna radiate) that show the influence of variety on harvest index. Spacing of $50 \times 30 \mathrm{~cm}$ and $50 \times 20 \mathrm{~cm}$ were ideal since they had a better harvest index than $50 \times 10 \mathrm{~cm}$ in all seasons. An increase of HI over $9 \%$ under wider spacing could be due to limited competition for growth factors that led to more assimilates partitioned into seed grain. Similarly, Mekuanint et al. (2018) reported an index of $53.25 \%$ under wider spacing. The $50 \times 10 \mathrm{~cm}$ spacing posted the lowest index in all seasons indicating the area cannot support denser populations that promote vegetative growth at the expense of grain yield. Similarly for other legumes, Kerina et al. (2017), and Agajie (2014) reported significantly lower harvest indices under small spacing than higher spacing in cowpea, Lablab, common bean, and chickpea.

Application of $\mathrm{N}_{0}$ and $\mathrm{N}_{30}$ rates generated the highest harvest index of $44 \%$ and $43 \%$ in LR2017 that could be attributed to the interaction with overall environmental conditions such as heat stress during the reproductive stage enhancing dry matter allocation into the seed (Muruiki et al. 2018). The results are in agreement with Doaiy et al. (2019), whose reported significant effects on yield and yield components under high nitrogen fertilization. Tamagno et al. (2018) established similar findings in Glycine max production. However, the highest harvest index value of $50 \%$ under $\mathrm{N}_{60}$ treatment in all seasons, indicates it was the best-suited N-rate for chickpea production under the study. Related findings were noted by Onyari et al. (2010), Wnuk et al. (2013), and Ndukhu et al. (2017) those who reported significant improvement in harvest index under nitrogen application. However, Seval et al. (2020) reported a shrinking harvest index in chickpea varieties under $\mathrm{N}$-application.

Interactive effects indicate that a combination of two or more factors simultaneously affects the outcome of a chickpea crop or cropping system yield (Onyari et al. 2010). Enhanced plant growth under wider spacing was due to less competition for nutrients and water (Wafula et al. 2021; Onyari et al. 2010). Muruiki et al. (2021) also reported differences in plant height among chickpea varieties ranging from $35 \mathrm{~cm}$ to $53 \mathrm{~cm}$. However, Mekuanint et al. (2018) noted non-significant results in height among chickpea 
varieties. Some varieties of chickpea are indeterminate, thus, growing after the vegetative stage. Indeterminate growth prolongs the reproductive period, which could be associated with higher plant height (citation). Interactions of Mwanza 2 x 50x20cm gave the highest biomass $\left(10.6 \mathrm{tha}^{-1}\right)$ over the study period, suggesting that increase in plant population likely increased biomass. However, Mwanza 2 and Chaina I x 50 x $30 \mathrm{~cm}$ spacing, gave better grain yield than other varieties showing that high biomass did not translate to high grains in all varieties. Saina $\mathrm{K}$ and Chaina III $x 50 \times 20 \mathrm{~cm}$ interaction also gave a higher yield than others did. High yield is due to the efficient utilization of growth factors under various interactions (Tehulie and Yimam 2021). The highest harvest index was noted under interactions of all varieties $\mathrm{x}$ $50 \times 30 \mathrm{~cm}$. Similarly, significant interactions between variety $\mathrm{x}$ spacing on chickpea heights, biomass, grain yield, and HI have been reported (Abdullah et al. 2019). This might be due to crop population, and adaptability to environmental conditions. Consistently across the observed traits, biomass decreased with increased spacing while grain yield increased with increased spacing. This implied that an increase in plants population led to an improvement in chickpea biomass that did not translate to high grain yields.

Interactions between varieties and nitrogen on measured parameters revealed significant results. Each variety improved height at different levels of nitrogen, with Mwanza 2 giving the highest heights under the $\mathrm{N}_{90}$ rate at all days of observation. Further, the observed interactive effects of variety and nitrogen (SN) on measured traits could be due to varied competitive capacity under spacing rates and nitrogen release. This conforms with the findings of Amiri et al. (2021) in soybeans that established heights of $64.26 \mathrm{~cm}$ to $97 \mathrm{~cm}$ under different $\mathrm{N}$-rates. Other findings are by Purushothaman et al. (2014), Naderi et al. (2021) and Basal and Szabó (2020) in chickpea are also in agreement with the findings of the present study. In contradiction, Pasqualone et al. (2021) established a higher mean plant in Desi $(79.39 \mathrm{~cm})$ than Kabuli $(68.83 \mathrm{~cm})$ under various nitrogen treatments $(30,40$, $\left.100 \mathrm{Kg} \mathrm{N} \mathrm{ha}^{-1}\right)$. Although Desi varieties expressed lower biomass than Kabuli, both gave low biomass under $\mathrm{N}_{0}$, but their maximum varied under various nitrogen treatments. The reverse was noted under harvest index with Desi (Chaina $\left.\mathrm{IxN}_{0}\right)$ expressing the highest harvest index (37\%) compared to other varieties, while Kabuli varieties (Mwanza 2 and Saina $\mathrm{K}$ ) $\mathrm{xN}_{0}$ performing lowest. Further, the findings of Devi et al. (2019) and Lemma et al. (2013), also established increased grain yield under variety $\mathrm{x}$ nitrogen fertilizer treatments than control. Finally, the joint interactive effects of variety, spacing, and nitrogen (VSN) application factor underscore the importance of joint consideration of the above factors on promoting chickpea production. The interaction between variety, spacing, and fertilizer application was significant on all the measured variables except for grain yield and harvest index in the
LR2018. The significant seasonal and treatment (variety, spacing, and nitrogen application rates) interactions on chickpea performance are due to environmental differences including rainfall amounts. The study findings were consistent with previous studies of Kaloki et al. (2019b) that reported significant effects between variety, environmental, and management interactions.

\section{Conclusion}

From the results, Kabuli varieties performed better in the study area than Desi varieties. Under variety $\mathrm{x}$ spacing interactions, Saina Kx50x10cm, Mwanza 2, Chaina I, and Chaina IIIx50x30cm were ideal for plant height. All varieties $\mathrm{x} 50 \mathrm{x} 10 \mathrm{~cm}$ gives high biomass, while $50 \times 30 \mathrm{~cm}$ was appropriate for high grain yield and harvest index. Varietyx $\mathrm{N}_{60}$ encouraged high plant height, biomass, and grain yield across most of the varieties, though Mwanza 2 was best under $\mathrm{N}_{90}$. All varieties improved harvest index with increasing $\mathrm{N}$ level except Chaina $\mathrm{I}$, which attained its maximum at $\mathrm{N}_{0}$. It is therefore important to note the following interactions enhanced grain yield, Saina $\mathrm{K} \times 50 \times 20 \mathrm{cmxN}_{90}$, Mwanza $2 \times 50 \times 30 \mathrm{~cm} \times$ N90, Chaina $\operatorname{Ix} 50 \times 30 \mathrm{cmxN}_{0}$, and Chaina IIIx $50 x 10 \mathrm{cmxN}_{30}$. To increase harvest index, all varieties to grow under $50 \times 30 \mathrm{cmxN}_{60}$ interactions, except Chaina I. However, narrow spacing is recommended for biomass production. Further research can be conducted for similar and other environmental factors using these varieties to validate findings.

\section{Conflicts of Interest}

The authors declare that they have no conflict of interest.

\section{Acknowledgements}

The authors are thankful to Kenya Agricultural, Livestock Research Centre (KALRO), and the University of Embu for supporting this work through soil sampling and analysis for this study. The authors also thank the Ministry of Agriculture for their support and encouragement throughout the study period.

\section{References}

Abebe, G.E.D.E.F.A.W., \& Debebe, S.I.S.A.Y. (2020). Factors Affecting chickpea Market Chain: The Case of Dembia District, North Gondar Zone, Ethiopia. Transportation P, 66.

Abdullah, S.A., Mohammed, B.I., \& Salih, R.H. (2019). Effect of Plant Spacing on Yield and Some Quality Characteristics of Three Chickpea (Cicer arietinum L.) Varieties. Polytechnic Journal, 9(1), 37-42. https://doi.org/10.25156/ptj.v9n1y2019.pp37-42.

Agajie, M. (2014). Effect of spacing on yield components and yield of chickpea (Cicer arietinum L.) at Assosa, Western Ethiopia. Agriculture, Forestry and Fisheries, 7(2), 39-51. 
Alemayehu, Y., Mieso, K., \&Ararsa, B. (2015). Effect of IntraRow Spacing on Haricot Bean (Phaseolus vulgaris L.) Production in humid Tropics of Southern Ethiopia. Journal of Natural Sciences Research, 5(15), 79-84.

Alemu, B., Abera, D., Adugna, A., \& Terefe, M. (2014) Adaptation Study of Improved Kabuli Chickpea (Cicer arietinum L) Varieties at Kellem Wollega Zone, Haro Sabu, Ethiopia. Journal of Natural Sciences Research 4(18), 21-24.

Amiri, H., Ghalavand, A., \& Mokhtassi-Bidgoli, A. (2021). Growth, seed yield and quality of soybean as affected by integrated fertilizer managements and zeolite application. Communications in Soil Science and Plant Analysis, 52(15), 1834-1851.

Arif, A., Parveen, N., Waheed, M.Q., et al. (2021). A comparative study for assessing the drought-tolerance of chickpea under varying natural growth environments. Frontiers in plant science, $11,2228$.

Banik, P., Midya, A., Sarkar, B.K., \& Ghose, S.S. (2006). Wheat and chickpea intercropping systems in an additive series experiment: advantages and weed smothering. European Journal of agronomy, 24(4), 325-332.

Basal, O., \& Szabó, A. (2020). The combined effect of drought stress and nitrogen fertilization on soybean. Agronomy, 10(3), 384.

Berrada, A.F., Shivakumar, B.G., \& Yaduraju, N.T. (2007). Chickpea in cropping systems. Chickpea breeding and management (pp.193).CABI publications.

Bhardwaj, H. L., \& Hamama, A. A. (2015). Cultivar, planting date, and row spacing effects on mungbean performance in Virginia HortScience, 50(9), 1309-1311.

Caliskan, S., Ozkaya, I., Caliskan, M.E., \& Arslan, M. (2008). The effects of nitrogen and iron fertilization on growth, yield and fertilizer use efficiency of soybean in a Mediterranean-type soil. Field Crops Research, 108(2), 126-132.

Dar, J.S., Pushpa, M.I.A.R., Abbassi, Z.A., \& Magsi, A.G. (2021). Effect of starter nitrogen on yield and yield components of chickpea (Cicer arietinum L.) at Dokri, Larkana. Pure and Applied Biology, 5(4), 1296-1303.

Devasirvatham, V., \& Tan, D.K. (2018). Impact of high temperature and drought stresses on chickpea production. Agronomy, 8(8), 145. https://doi.org/10.3390/agronomy8080145.

Devi, P.B., Darvhankar, M.S., Prakash, A., \& Banik, D. (2019). Effect of different doses of nitrogen on growth and yield of Chickpea (Cicer arietinum). Plant Archives, 19(1), 458-460.
Dhima, K., Vasilakoglou, I., Stefanou, S., \& Eleftherohorinos, I. (2015). Effect of cultivar, irrigation and nitrogen fertilization on chickpea (Cicer arietinum L.) productivity. Agricultural Sciences 6(10):1187-1194.

Doaiy, F., NakhzariMoghaddam, A., RahemiKarizaki, A., \& Aldaghi, M. (2019). Chemical and biological fertilizer management of nitrogen effects on yield and yield components of chickpea (Cicer arietinum L.) in different planting dates. Iranian Journal Pulses Research, 10(1), 28-39.

FAOSTAT FAO (2019). Food and Agriculture Organization of the United Nations-Statistic Division Retrieved from https://www. fao. org/faost at/en/\# data.

Fazle, B.U., Sultana, B.S., Jahan, M.S., \& Paul, A.K. (2014). Effect of different levels of nitrogen and phosphorus on the growth and yield of garden pea (Pisumsativum L.). Eco-friendly Agriculture Journal 7(09):93-99.

Fikre, A., Desmae, H., \& Ahmed, S. (2020). Tapping the economic potential of chickpea in sub-Saharan Africa. Agronomy, 10(11), 1707. https://doi.org/10.3390/agronomy10111707.

Gaur, P.M., Tripathi, S., Gowda, C.L.L., et al. (2010). Chickpea Seed Production Manual. Patancheru 502 324, Andhra Pradesh, India: International Crops Res. International Crops Research Institute for the Semi-Arid Tropics. 28 pp. 2010. ICRISAT. Retrieved from http://oar.icrisat.org/8809/1/India\%20Chickpea\% 20Production.pdf.

Gezahegn, A.M., Tesfaye, K., Sharma, J.J., \& Belel, M.D. (2016). Determination of optimum plant density for faba bean (Viciafaba L.) on vertisols at Haramaya, Eastern Ethiopia. Cogent Food \& Agriculture, 2(1), $1224485 . \quad$ https://doi.org/10.1080/ 23311932.2016 .1224485 .

Goa, Y. (2014). Evaluation of chickpea (Cicer arietinum L.) varieties for yield performance and adaptability to southern Ethiopia. Journal of Biology, Agriculture and Healthcare, 4(17), 34-38.

Goa, Y., \& Ashamo, M. (2016). Yield Performance and Adaptation of Desi Chick Pea Varieties in Selected Districts of Wolayta and Hadiya Zones of South Ethiopia. International Journal of Research-Granthaalayah, 4(3), 33-41.

Hossain, Z., Wang, X., Hamel, C., et al. (2016). Biological nitrogen fixation by pulse crops on semiarid Canadian prairies. Canadian Journal of Plant Science, 97(1), 119-131. https://doi.org/10.1139/cjps-2016-0185. 
Jaetzold, R., Schmidt, H. (1982). Farm Management Handbook of Kenya: Natural conditions and farm management information / by Ralph Jaetzold and Helmut Schmidt. Central Kenya (Rift Valley and Central Provinces), Volume 2. Kenia Ministry of Agriculture https://www.yumpu.com/en/document/view/41630926.

Kaloki, P., Trethowan, R., \& Tan, D.K. (2019a). Effect of genotypex environment× management interactions on chickpea phenotypic stability. Crop and Pasture Science, 70(5), 453-462.

Kaloki, P., Devasirvatham, V., \& Tan, D.K. (2019b). Chickpea abiotic stresses: combating drought, heat and cold. In de Oliveira, A.B. (ed) Abiotic and biotic stress in plants. IntechOpen Book series DOI: 10.5772/intechopen.83404.

Kalungu, J.W., \& Harris, D. (2013). Smallholder farmers' perception of the impacts of climate change and variability on rainfed agricultural practices in semi-arid and sub-humid regions of Kenya. Journal of Environment and Earth Science, 3(7), 129-140.

Kamithi, D.K., Kibe, A.M., \& Akuja, T.E. (2009). Effects of nitrogen fertilizer and plant population on growth, yield and harvest index (HI) of chickpea (Cicer arietinum L.) under dryland conditions in Kenya. Journal of Applied Biosciences, 22, 13591367.

Keerthi, M.M., Babu, R., Joseph, M., \& Amutha, R. (2015). Optimizing plant geometry and nutrient management for grain yield and economics in irrigated greengram. American Journal of Plant Sciences, 6(08), 1144.

KMD (2018). Kenya Meteorological Weather forecast report.Mbeere South, Embu County.

KMD (2017). Kenya Meteorological Weather forecast report.Mbeere South, Embu County.

Kerina, A., Ngode, L., Opile, W., \& Mwamburi, L. (2017). Response of Three Legumes to Inorganic Fertilizer Application in Different Agro-Ecological Zones in Makueni County Kenya. African Journal of Education, Science and Technology, 4(1), 1425 .

Khan, E.A., Aslam, M., Ahmad, H.K., Ayaz, M., \& Hussain, A. (2010). Effect of row spacing and seeding rates on growth yield and yield components of chickpea. Sarhad Journal of Agriculture, 26(2), 201-211.

Khaitov, B., \&Abdiev, A. (2018). Performance of chickpea (Cicer arietinum L.) to bio-fertilizer and nitrogen application in arid condition. Journal of Plant Nutrition, 41(15), 1980-1987.
Kherif, O., Seghouani, M., Zemmouri, B., et al. (2021). Understanding the Response of Wheat-Chickpea Intercropping to Nitrogen Fertilization Using Agro-Ecological Competitive Indices under Contrasting Pedoclimatic Conditions. Agronomy, 11(6), 1225. https://doi.org/10.3390/agronomy11061225.

Kiboi, M.N., Ngetich, K.F., Fliessbach, A., Muriuki, A., \& Mugendi, D.N. (2019). Soil fertility inputs and tillage influence on maize crop performance and soil water content in the Central Highlands of Kenya. Agricultural Water Management, 217, 316331.

Kimiti, J.M., Odee, D.W., \& Vanlauwe, B. (2009). Area under grain legumes cultivation and problems faced by smallholder farmers in legume production in the semi-arid eastern Kenya. Journal of Sustainable Development in Africa, 11 (4), 305-315.

Lemma, W., Wassie, H., \& Sheleme, B. (2013). Response of chickpea (Cicer arietinum L.) to nitrogen and phosphorus fertilizer $\mathrm{S}$ in halaba and taba, Southern Ethiopia. Ethiopian Journal of Natural Resources, 13(2), 115-128.

Księżak, J., \& Bojarszczuk, J. (2020). The effect of cropping method and botanical form on seed yielding and chemical composition of chickpeas (Cicer arietinum L.) grown under organic system. Agronomy, 10(6), 801. https://doi.org/10.3390/ agronomy10060801.

Li, J., Huang, L., Zhang, J., et al. (2019). Diversifying crop rotation improves system robustness. Agronomy for Sustainable Development, 39(4), 1-13. https://doi.org/10.1007/s13593-0190584-0.

Li, L., Yang, T., Redden, R., He, W., \& Zong, X. (2016). Soil fertility map for food legumes production areas in China. Scientific Reports, 6(1), 1-14. https://doi.org/10.1038/srep26102.

Macák, M., Candráková, E., Đalović, I., et al. (2020). The Influence of Different Fertilization Strategies on the Grain Yield of Field Peas (Pisum sativum L.) under Conventional and Conservation Tillage. Agronomy, 10(11), 1728. https://doi.org/10.3390/agronomy10111728.

Mallu, T.S. (2015). Evaluation of Chickpea Genotypes for Yield and Selected Agronomic Traits in Kenya. Ph.D. dissertation submission to College of Engineering and Technology, Jomo Kenyatta University of Science and Technology, Nairobi, Kenya. Retrieved from https://ir.jkuat.ac.ke/handle/123456789/1699.

Maya, M., \& Maphosa, M. (2020). Current status of chickpea production: Opportunities for promoting, adoption and adapting the crop in Zimbabwe: A review. Journal of Dryland Agriculture, $6(1), 1-9$. 
McVay, K.A., \& Crutcher, F. (2011). Chickpea production. Retrieved from https://pspp.msuextension.org/documents/ Chickpea\%20Production.pdf.

Mekuanint, T., Tsehaye, Y., \& Egziabher, Y.G. (2018). Response of two chickpea (Cicer arietinum L.) varieties to rates of blended fertilizer and row spacing at Tselemti district, northern Ethiopia. Advances in Agriculture, Article ID 5085163. https://doi.org/10.1155/2018/5085163.

Merga, B., \& Haji, J. (2019). Economic importance of chickpea: Production, value, and world trade. Cogent Food \& Agriculture, 5(1), 1615718.

Ministry of agriculture, MoA. (2013). Crops extension pocket handbook revised edition: Field crops 1.

Mousavi, S.K. (2019). Wild barley (Hordeum spontaneum C. Koch) response to weed management approaches in wheatchickpea-wheat rotation. Iranian Journal of Weed Science 15(1):125-38.

Muehlbauer, F.J., \& Sarker, A. (2017). Economic Importance of Chickpea: Production, Value, and World Trade. In: Varshney, R., Thudi, M., Muehlbauer F. (eds) The Chickpea Genome. Compendium of Plant Genomes. Springer, Cham. https://doi.org/10.1007/978-3-319-66117-9_2

Muruiki, R., Kimurto, P., Towett, B., \& Rao, G. (2021). Yield performance of chickpea (Cicer arietinum L.) genotypes under supplemental irrigation regimes in semi-arid tropics. Journal of Plant Breeding and Crop Science, 13(4), 177-189.

Muruiki, R., Kimurto, P., Vandez, V., et al. (2018). Effect of drought stress on yield performance of parental chickpea genotypes in semi-arid tropics. Journal of Life Sciences, 12(3), 159-168.

Naderi, R., Bijanzadeh, E., \& Egan, T.P. (2021). Short-term Response of Chickpea Yield, Total Soil Carbon, and Soil Nitrogen to Different Tillage and Organic Amendment Regimes. Communications in Soil Science and Plant Analysis, 52(9), 9981007.

Namvar, A., Sharifi, R.S., \& Khandan, T. (2011). Growth analysis and yield of chickpea (Cicer arietinum L.) in relation to organic and inorganic nitrogen fertilization. Ekologija, 57(3), 97-108.

Ndukhu, O., Onwonga, N., Wahome, G., Kironch, G., \& Høgh Jensen, H. (2017). Effect of Integrating Chickpea and Organic Amendments on Yield and Quality Attributes of Tomato and Maize in Central Kenya. Journal of Agriculture and Ecology Research International, 12(4), 1-22.
Ngetich, K.F., Diels, J., Shisanya, C.A., et al. (2014). Effects of selected soil and water conservation techniques on runoff, sediment yield and maize productivity under sub-humid and semiarid conditions in Kenya. Catena, 121, 288-296.

Ogola, J.B.O., Mathews, C., \& Magongwa, S.M. (2013). The productivity of cassava-legume intercropping system in a dry environment in Nelspruit, South Africa. In African Crop Science Conference Proceedings, 11, 61-65.

Onyari, C.N., Ouma, J., \& Kibe, A.M. (2010). Effect of tillage method and sowing time on phenology, yield and yield components of chickpea (Cicer arietinum L.) under semi-arid conditions in Kenya. Journal of Applied Biosciences, 34, 2156 2165 .

Pasqualone, A., Summo, C., De Angelis, D., et al. (2021). Effect of Mineral and Organic Fertilization on desi and kabuli Chickpea (Cicer arietinum L.): Plant Growth and Production, Hydration Properties, Bioactive Compounds, and Antioxidant Activity. Plants, 10(7), 1441.

Patil, S.H., Wadikar, P.B., Dhutraj, D.N., \& Sargar, P.R. (2021). Correlation Analysis for Grain Yield and its Components in Pearl Millet [Pennisetumglaucum (L.) R. Br.]. Madras Agricultural Journal, 108(4-6), 1.

Purushothaman, R., Upadhyaya, H.D., Gaur, P.M., Gowda, C.L.L., \& Krishnamurthy, L. (2014). Kabuli and desi chickpeas differ in their requirement for reproductive duration. Field Crops Research, 163, 24-31. https://doi.org/10.1016/j.fcr.2014.04.006.

Raimi, A., Adeleke, R., \& Roopnarain, A. (2017). Soil fertility challenges and Biofertiliser as a viable alternative for increasing smallholder farmer crop productivity in sub-Saharan Africa. Cogent Food \& Agriculture, 3(1), 1400933. https://doi.org/10.1080/23311932.2017.1400933.

Rao, N.V. P.R.G., Silim, S.N., Simtowe, F., et al. (2012). Enhancing chickpea productivity and production in eastern and southern Africa. ICRISAT Report, Progress of Phase 1:177-190. Retrieved from https://oar.icrisat.org/6616/.

Richards, M., Maphosa, L., Preston, A., Napier, T., \& Hume, I. (2019). Pulse adaptation-optimising grain yield of chickpea and lentils. Retrieved from https://grdc.com.au/_data/assets/ pdf_file/0037/399664/Paper-Richards-Mark-et-al-Wagga-Update2020.pdf.

Rutherford, A. (2011). ANOVA and ANCOVA: a GLM approach. John Wiley \& Sons. 
SAS, B. (2015). Base SAS 92 Procedures Guide Pdf. Retrieved from https://documentation.sas.com/doc/en/pgmsascdc/9.4_3.5/.

Sellami, M.H., Lavini, A., \& Pulvento, C. (2021). Phenotypic and quality traits of chickpea genotypes under rainfed conditions in south Italy. Agronomy, 11(5), 962.

Seval, E.L.İ.Ş., İpekeşen, S., Başdemir, F., Murat, T.U.N.Ç., \& Bicer, B. (2020). Effect of different fertilizer forms on yield and yield components of chickpea varieties. International Journal of Agriculture Environment and Food Sciences, 4(2), 209-215.

Schilt-van Ettekoven, C., Giller, K.E., Ton, G., Glover, D.B.A., et al. (2020). N2Africa Podcaster no. 58: Putting nitrogen fixation to work for smallholder farmers in Africa: January 2020 (No. 58). N2Africa project. Retrieved from https://n2africa.org/sites/default/ files/N2Africa\%20Podcaster\%2058.pdf.

Shumi, D., Alemayehu, D., \& Afeta, T. (2020). Adaptation Study of Improved Chickpea (Cicer arietinum L.) Varieties at Mid and highland of Guji zone, Southern Ethiopia. Journal of Agricultural Science and Research, 6(1), 42-46.

Sims, L., Pastor, J., Lee, T., \& Dewey, B. (2012). Nitrogen, phosphorus and light effects on growth and allocation of biomass and nutrients in wild rice. Oecologia, 170(1), 65-76.

Tamagno, S., Sadras, V.O., Haegele, J.W., Armstrong, P.R., \& Ciampitti, I.A. (2018). Interplay between nitrogen fertilizer and biological nitrogen fixation in soybean: implications on seed yield and biomass allocation. Scientific reports, 8(1), 1-11.

Tamiru, T., Worku, W., Mengesha, W., \& Weyya, G. (2020). Response of Chickpea (Cicer aeritinum L.) Genotype to Intra and Inter Row Spacing at HuletegnaChoroko, Southern Ethiopia. Ecology and Evolutionary Biology, 5(4), 148 https://doi.org/10.11648/j.eeb.20200504.15.

Tuarira, M., \& Moses, M. (2014). Effects of plant density and planting arrangement in green bean seed production. Journal of Global Innovations in Agriculture and Social Sciences, 2(4), 152-
157. https://doi.org/10.17957/JGIASS/2.4.516.

Tehulie, N.S., \& Yimam, K.M. (2021). Review on the Effect of Plant Population on Yield and Yield Components of Chickpea (Cicer arietinum L.). Journal of Agricultural Research Pesticides and Biofertilizers, 1(5), 46-49. DOI:http;//doi.org/05.2021/1.1024.

Uchhara, C.P., Onyeonagu, C.C., \& Asiegbu, J.E. (2013). Effect of plant population and $\mathrm{N}$ fertilizer on the growth and yield of bambara groundnut (Vigna subterranean (L.) Verdc.). AgroScience, 12(3), 25-34.

Uddin, M., Hussain, S., Khan, M.M.A., et al. (2014). Use of N and $\mathrm{P}$ biofertilizers reduces inorganic phosphorus application and increases nutrient uptake, yield, and seed quality of chickpea. Turkish Journal of Agriculture and Forestry, 38(1), 47-54.

Under Newly, S.F.B.V. (2011). Effect of row spacing on yield and its components of some faba bean varieties under newly reclaimed sandy soil condition. World Journal of Agricultural Sciences, 7(1), 68-72.

Vaghar, M.S., Kobraee, S., Shamsi, K., \& Behrooz, R. (2013). The economic yield evaluation and some of the morphological traits of chickpea cultivars under the influence of different densities. International Journal of Biosciences, 3(12), 232-244. https://dx.doi.org/10.12692/ijb/3.12.232-244.

Wafula, J.K., Njoka, F.M., Nthakanio, P.N., et al. (2021). Nodulation and protein content of selected chickpea varieties as impacted by spacing and n-fertiliser rates in Eastern Kenya. African Journal of Agricultural Research, 17(6), 882-895. https://doi.org/10.5897/AJAR2021.15597.

Wallace, T.C., Murray, R., \& Zelman, K.M. (2016). The nutritional value and health benefits of chickpeas and hummus. Nutrients, 8(12), 766. doi: 10.3390/nu8120766.

Wnuk, A., Górny, A.G., Bocianowski, J., \& Kozak, M. (2013). Visualizing harvest index in crops. Communications in Biometry \& Crop Science, 8(2), 48-59. 